\title{
Multi-quark colour decompositions from unitarity
}

\author{
Alexander Ochirov ${ }^{a}$ and Ben Page ${ }^{b}$ \\ ${ }^{a}$ ETH Zürich, Institut für Theoretische Physik, \\ Wolfgang-Pauli-Str. 27, 8093 Zürich, Switzerland \\ ${ }^{b}$ Institut de Physique Théorique, CEA, CNRS, Université Paris-Saclay, \\ F-91191 Gif-sur-Yvette cedex, France \\ E-mail: aochirov@phys.ethz.ch, bpage@ipht.fr
}

\begin{abstract}
Any loop QCD amplitude at full colour is constructed from kinematic and gauge-group building blocks. In a unitarity-based on-shell framework, both objects can be reconstructed from their respective counterparts in tree-level amplitudes. This procedure is at its most powerful when aligned with flexible colour decompositions of tree-level QCD amplitudes. In this note we derive such decompositions for amplitudes with an arbitrary number of quarks and gluons from the same principle that is used to bootstrap kinematics - unitarity factorisation. In the process we formulate new multi-quark bases and provide closed-form expressions for the new decompositions. We then elaborate upon their application in colour decompositions of loop multi-quark amplitudes.
\end{abstract}

KEYwords: Perturbative QCD, Scattering Amplitudes

ArXiv EPRINT: 1908.02695 


\section{Contents}

1 Introduction 1

1.1 Example of colour constraints from unitarity 3

2 Colour and unitarity $\quad 4$

2.1 Colour factorisation 6

$\begin{array}{lll}2.2 & \text { Leg-exchange relation and colour-ordered splitting } & 7\end{array}$

3 Co-unitary tree-level bases and colour decompositions $\quad 9$

$\begin{array}{llr}3.1 & \text { Like-flavour stretch } & 9\end{array}$

$\begin{array}{ll}3.2 & \text { Distinct-flavour stretch } \\ 3.3 & \text { Stretching with }\end{array}$

$\begin{array}{lll}3.3 & \text { Stretching with gluons } & 16\end{array}$

$\begin{array}{ll}3.4 & \text { Properties of tree-level bases } \\ & 3.4 .18\end{array}$

$\begin{array}{lll}3.4 .1 & \text { Co-unitarity } & 18\end{array}$

$\begin{array}{ll}3.4 .2 & \text { Colour-ordered splitting }\end{array}$

$\begin{array}{lll}3.4 .3 & \text { Linear independence } & 22\end{array}$

4 Loop-level applications $\quad 23$

5 Summary and outlook $\quad 28$

$\begin{array}{lr}\text { A Colour Feynman rules and colour ordering } & 29\end{array}$

$\begin{array}{ll}\text { B Colour-unitarity checks } & 29\end{array}$

\section{Introduction}

The improving precision of the experimental measurements of multi-jet processes at the Large Hadron Collider has motivated an array of new theoretical results for QCD scattering amplitudes with more than two partons in the final state [1-13]. In particular, the first non-planar five-point two-loop amplitude has been recently computed for pure Yang-Mills theory in ref. [13], starting from the full-colour integrand of ref. [14]. This remarkably simple result for the five-gluon amplitude with all helicities chosen positive reflects the known structure at the integrand level [14-16].

The demand for accuracy will inevitably require going beyond the leading-colour approximation at two loops. Furthermore, many relevant two-loop observables, such as Higgs plus jets, have non-planar contributions even in this approximation, and so a thorough understanding of the gauge-group degrees of freedom is essential. On-shell methods, i.e. based on unitarity cuts [17-19], are a promising approach to compute such higher-multiplicity two-loop amplitudes, and the loop-colour method of refs. $[14,20]$ provides a systematic 
on-shell method for handling colour information at the multi-loop level. In a nutshell, the method consistently retains the colour factors of the tree amplitudes of generalised unitarity cuts when reconstructing the loop integrand. It was detailed in ref. [20] with a focus on the case of purely adjoint-representation particle content.

A key ingredient for the loop-colour method presented in ref. [20] was a flexible treelevel colour decomposition. In the adjoint case, the method relies on the decomposition of del Duca, Dixon and Maltoni (DDM) [21]. This decomposition is a "proper" colour decomposition - it splits the colour and kinematic degrees of freedom in such a way that the kinematic objects are linearly independent planar ordered amplitudes, in which only factorisation channels with consecutively ordered particles may appear (see e.g. ref. [22]). More precisely, the DDM decomposition expresses a purely gluonic amplitude in terms of a basis of $(n-2)$ ! ordered amplitudes independent under Kleiss-Kuijf (KK) relations [23] where the associated colour factors are given by strings of the structure constants

$$
\begin{aligned}
& \mathcal{A}_{n}^{\text {tree }}=\sum_{\sigma \in S_{n-2}} \tilde{f}^{a_{1} a_{\sigma(2)} b_{1}} \tilde{f}^{b_{1} a_{\sigma(3)} b_{2}} \ldots \tilde{f}^{b_{n-4} a_{\sigma(n-2)} b_{n-3}} \tilde{f}^{b_{n-3} a_{\sigma(n-1)} a_{n}} \\
& \times A(1, \sigma(2), \ldots, \sigma(n-1), n)
\end{aligned}
$$

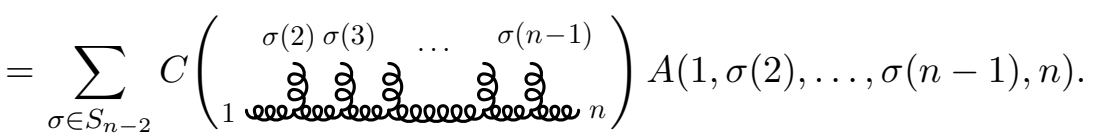

Note that in the ordered amplitudes the positions of gluons 1 and $n$ are fixed next to each other, and the topology of the corresponding colour factors can be understood as "stretched between" these gluons. A similar colour decomposition is known for tree amplitudes with an arbitrary number of quark lines $[24,25]$ but restricted to the basis in which two quarks of the same flavour are fixed next to each other $[26,27]$.

In this paper we give new tree-level colour decompositions for multi-quark amplitudes, which allow any pair of particles to be fixed next to each other in the ordered-amplitude sum. These decompositions are also "proper", sharing the crucial property of the DDM formula (1.1): the constituent ordered amplitudes are independent with respect to the KK relations. Importantly, we find that once such bases of ordered amplitudes are found, the colour factors in these decompositions are easily fixed by imposing the principle of kinematic factorisation. Remarkably, the colour factors in such decompositions obey factorisation relations themselves. This then transparently explains the "stretched" nature of the colour factors in the DDM decomposition (1.1), a feature inherited by all of the new decompositions in this work. When inserted into unitarity cuts, these results allow one to realise the full power of the loop-colour method [20] from pure Yang-Mills theory to QCD.

Note that we only consider the colour algebra defined with the most generic set of relations - the Jacobi and commutation relations - avoiding the details of the kinematic amplitude dependence. Therefore, all our results are not specific to quarks but hold for other gauged matter particles in arbitrary representations of the gauge group, whose flavour is conserved in gauge interactions. ${ }^{1}$

\footnotetext{
${ }^{1}$ In other words, each flavoured colour-flow line may contain its own generators. For example, what we refer to here as the quark line $\underline{1} \leftarrow \overline{2}$ could just as well be a complex scalar in the antifundamental representation of $\mathrm{U}\left(N_{\mathrm{c}}\right)$, meaning that its actual charge would flow against the arrow.
} 
The bulk of this paper is structured in a general-to-detailed way. First, in section 2, we describe the main result of this paper - KK-independent tree-level colour decompositions for an arbitrary stretch, whose colour factors satisfy a set of recursion relations. Then in section 3 we specify the main technical advance allowing these decompositions: a collection of "co-unitary" KK-independent bases of tree-level amplitudes that obey certain factorisation properties. Next, in section 4, we outline their application at loop level within the method of ref. [20] and discuss the one-loop case [28-33] in some detail. Finally, we conclude by presenting our outlook in section 5 .

However, before we proceed in this way, let us illustrate the appropriateness of a unitarity-based approach to colour with a simple five-point example.

\subsection{Example of colour constraints from unitarity}

In this section we use an amplitude with two quark pairs and one gluon to show how a new colour decomposition can be constructed from factorisation properties. We label the first quark pair as $\underline{1}$ and $\overline{2}$, the second quark pair as $\underline{3}$ and $\overline{4}$, and the gluon as 5 . The previously known colour decomposition [24] would correspond to a $q \bar{q}$ stretch. Here we wish to consider a $q g$ stretch. Fixing for definiteness quark 1 and gluon 5 next to each other, let us look for a tentative colour decomposition

$$
\mathcal{A}_{5,2}^{\text {tree }}=C(\underline{1}, \overline{2}, \underline{3}, \overline{4}, 5) A(\underline{1}, \overline{2}, \underline{3}, \overline{4}, 5)+C(\underline{1}, \overline{2}, \overline{4}, \underline{3}, 5) A(\underline{1}, \overline{2}, \overline{4}, \underline{3}, 5)+C(\underline{1}, \underline{3}, \overline{4}, \overline{2}, 5) A(\underline{1}, \underline{3}, \overline{4}, \overline{2}, 5) .
$$

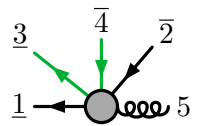

Here we give a graphic representation of the cyclic amplitude orderings, which will be used extensively throughout the paper. For brevity, we have simply assumed that the orderings above constitute a valid basis, although this can be argued from the presence of the physical factorisation channels just as well. For notational convenience in this paper we shall consider massless amplitudes, though this is not required for the results to hold.

To constrain the unknown colour factors in eq. (1.2), we consider the factorisation limits of the full amplitude on the poles that include gluon 5 and one of the unfixed quarks:
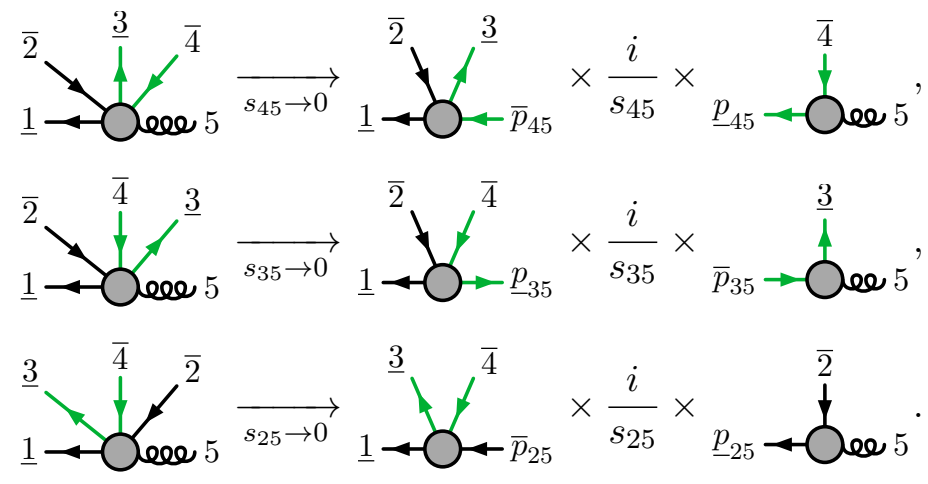

As each pole only appears in one ordered amplitude, the blob diagrams above can be regarded as either colour-dressed amplitudes (in which case those on the left-hand side 
are all $\left.\mathcal{A}_{5,2}^{\text {tree }}\right)$ or their ordered counterparts. Now recall the trivial decomposition of the four-point pure-quark amplitude ${ }^{2}$

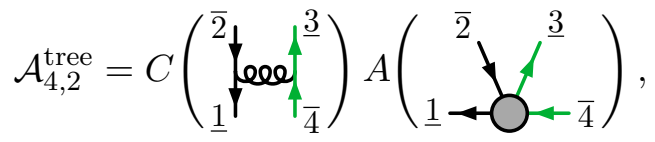

which must appear in the above factorisation limits of the five-point amplitude. Applying the limits (1.3) both to the five-point decomposition (1.2) as a whole and to the constituent ordered amplitudes therein, we can directly read off the colour coefficients as

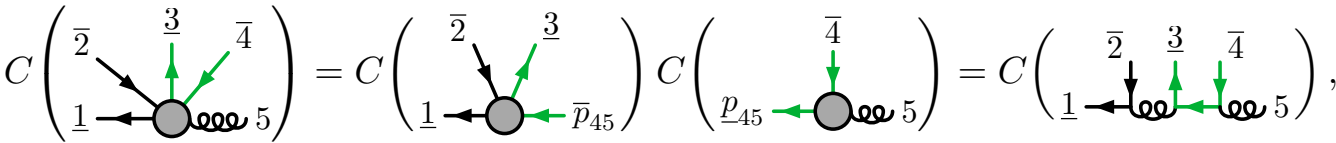

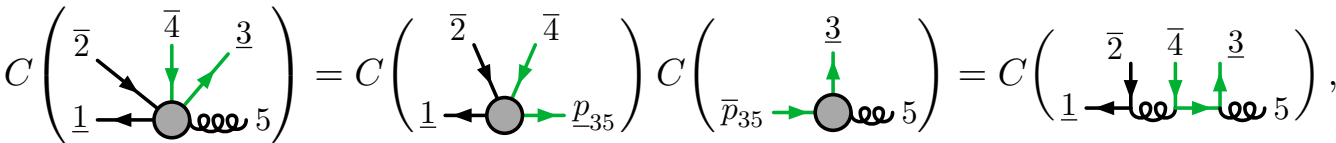

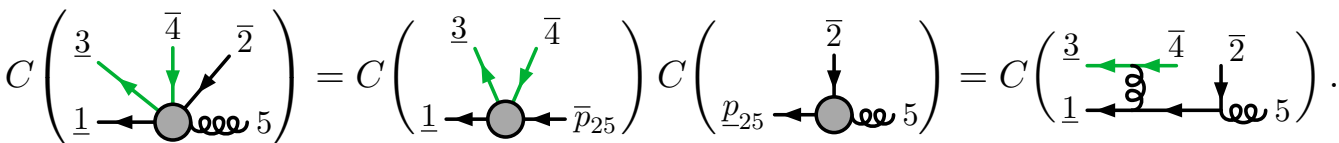

The kinematic limits here became identities, since of course the colour factors are independent of any momenta. In this way, we have used the factorisation properties of the amplitude to derive a new explicit colour decomposition

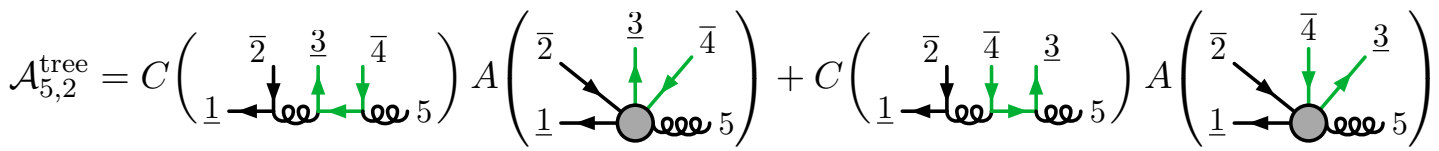

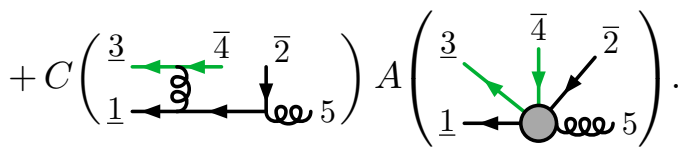

\section{Colour and unitarity}

The basic principle that we shall be employing in this work is that colour degrees of freedom play no kinematic role, and so the operations of performing a colour decomposition and taking a factorisation limit commute. This implies strong constraints on any colour decomposition, and we will show that, given a good choice of basis of ordered amplitudes, such constraints can be used to fix it entirely. We will prove the existence of a collection of proper colour decompositions of the form

$$
\mathcal{A}(1, X, n)=\sum_{\sigma \in \mathcal{B}_{X}^{1, n}} C\left(1 \stackrel{\sigma}{\sigma}{ }_{n}\right) A\left(1 \stackrel{\sigma}{\sigma_{n}}\right) .
$$

\footnotetext{
${ }^{2}$ In our notation, $C$ applied to a trivalent diagram (without blobs) translates to its colour factor obtained using the colour Feynman rules recalled in appendix A, whereas $C$ applied to a blob diagram means the colour coefficient of an ordered amplitude within the context of some colour decomposition. These notions coincide in the factorisation limits that expose the maximal number of propagators, as the colour coefficients of three-point amplitudes are always the same as the colour factors of the corresponding trivalent vertices.
} 
Here we portray particles 1 and $n$ as "stretched", and we sum over a KK-independent basis, indexed by the ordered tuples $\mathcal{B}_{X}^{1, n}$. This quite abstract depiction is due to the generality of these decompositions - particles 1 and $n$ can be taken arbitrarily. That is, we will present decompositions for distinct-flavour multi-parton tree-level amplitudes stretched across two gluons, a gluon and a quark, and two quarks (which may be distinct). This generalises the DDM decomposition [21] and that of ref. [24] to a new level of flexibility.

As we shall see, the colour factors in these decompositions can be entirely fixed by recursion relations obtained from factorisation constraints. Remarkably, we will show that the colour factors in our bases obey a type of factorisation, being given as products of colour factors of lower-point decompositions. Specifically, if we consider a colour factor in some decomposition where we have fixed particles 1 and $n$ to be next to each other, then

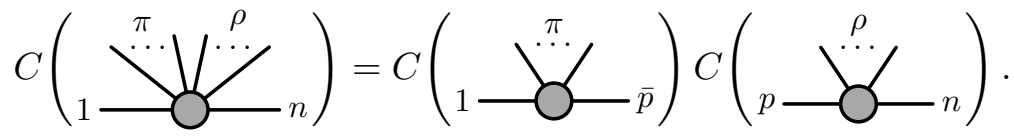

That is, the colour factor of the ordered amplitude $A(1, \pi, \rho, n)$, with $\pi$ and $\rho$ being ordered sets, factorises into the colour factors of the ordered amplitudes $A(1, \pi, \bar{p})$ and $A(p, \rho, n)$, where $p$ is the particle that allows the two factor amplitudes to conserve all charges. Importantly, $p$ may not exist, at which point eq. (2.2) does not apply. This may occur, for example, where there is no way for a single particle to conserve quark flavour.

The colour factorisation relation (2.2) alone is sufficient to fully fix the DDM decomposition. Indeed, as $p$ is always a gluon in the purely gluonic case, the colour factors in the DDM decomposition can be repeatedly split by eq. (2.2) until one arrives at a product of three-point colour factors - the familiar "comb" structure of eq. (1.1).

In the presence of matter particles, the abstract generality of the colour factorisation (2.2) is a consequence of a crucial property of the bases presented in this work (as well as the KK [23] and Melia bases [26, 27]), which we dub "co-unitarity". This highly nontrivial condition is that for every factorisation limit that separates particles 1 and $n$, the surviving terms are independent under KK relations. We define this notion more precisely, and write down a set of co-unitary bases in section 3. Furthermore, we will find that any set of colour-ordered amplitudes that satisfies this property is automatically a basis.

Beyond eq. (2.2), we shall show further relations between the factors of our decompositions, that completely fix the colour factors in terms of three-point colour factors. First, if there is a quark pair with the quark and its antiquark next to each other, then we find a "leg-exchange" relation,

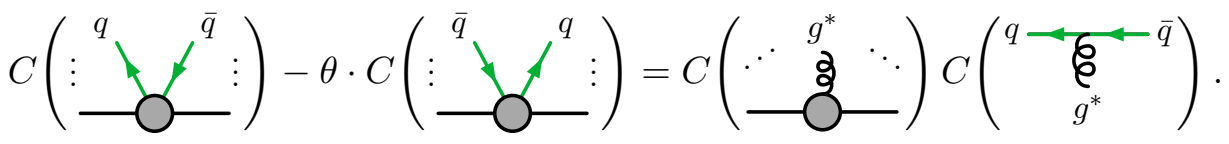

Here $\theta$ reflects the fact that the second term only contributes to the relation if both associated ordered amplitudes appear in a colour decomposition. As will be explained in detail in section 3 and is familiar from the Melia basis [26, 27], a given colour decomposition may not include both orderings of a quark pair. When only a single ordering is allowed, then $\theta=0$, otherwise $\theta=1$. Similarly, we will show that if we have a colour factor of an ordered 
amplitude where the gluon is on one side of an (anti)quark, we can move it to the other, at the price of generating an extra term:

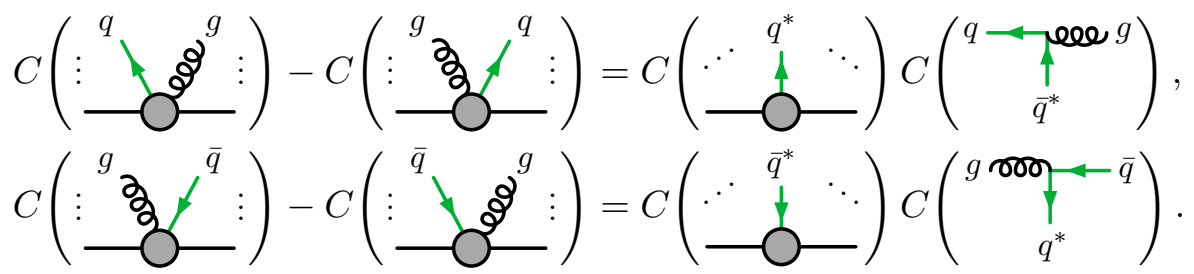

Finally, the colour factors of two ordered amplitudes that differ only by a permutation of two adjacent gluons differ by a similar term:

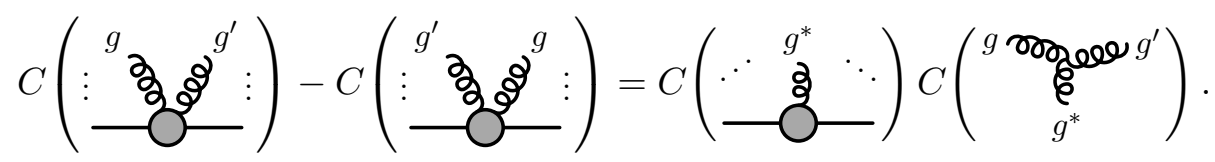

Naturally, these leg-exchange identities are a consequence of the Jacobi identity and commutation relations,

$$
\begin{aligned}
\tilde{f}^{d a c} \tilde{f}^{c b e}-\tilde{f}^{d b c} \tilde{f}^{c a e} & =\tilde{f}^{a b c} \tilde{f}^{d c e}, \\
T_{i \bar{\jmath}}^{a} T_{j \bar{k}}^{b}-T_{i \bar{\jmath}}^{b} T_{j \bar{k}}^{a} & =\tilde{f}^{a b c} T_{i \bar{k}}^{c} .
\end{aligned}
$$

However, we shall see that they can also regarded as a consequence of factorisation.

These relations are a consequence of requiring that the decompositions act trivially under various factorisation limits. In the following subsections we shall discuss these limits and the constraints that they put on the colour-ordered amplitudes in the bases. We shall later see in section 3 that these constraints can indeed be realised.

\subsection{Colour factorisation}

Let us now show that, given an appropriate basis of colour-ordered amplitudes, the colour factorisation relation (2.2) indeed follows from kinematic factorisation. We will consider an $n$-particle amplitude, privileging particles 1 and $n$ and partitioning the remaining particles into the unordered sets $P$ and $R$. We now consider a factorisation limit (implemented as a residue) on the channel collecting all particles 1 and $P$, and then colour-decompose the resulting product:

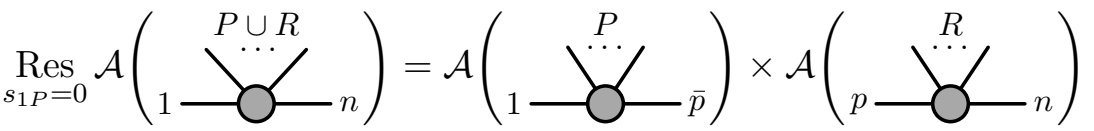

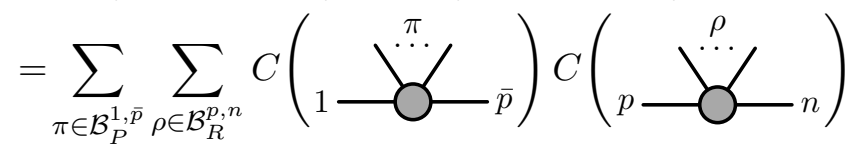

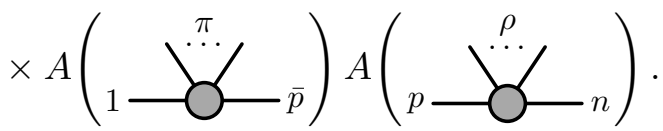

Here and below we use $\mathcal{A}$ to represent full-colour amplitudes, $A$ to represent colour-ordered amplitudes, and the notation $\mathcal{B}_{X}^{a, b}$ to indicate the permutation set corresponding to the basis of ordered amplitudes with particles $a$ and $b$ stretched across the set of particles $X$. 
Importantly, there are no (kinematically independent) relations between the ordered amplitudes on the right-hand side as the linear independence is inherited from the individual colour decompositions. In order to find a constraint, we next consider the opposite order of operations - we first perform colour decomposition and then take the factorisation limit:

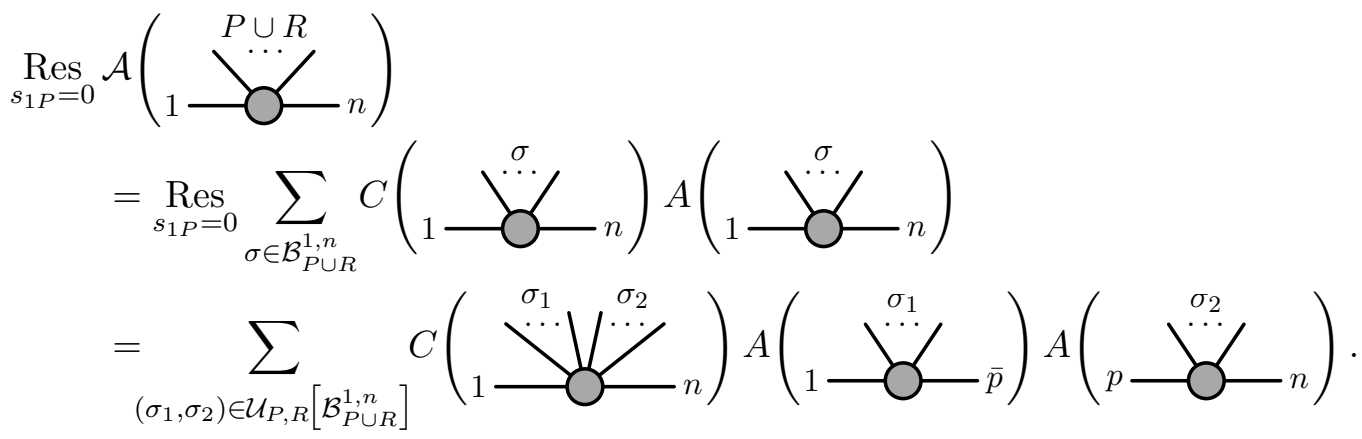

Here the sum is over all terms in the colour decomposition that have the chosen residue, so the implied definition for the set $\mathcal{U}_{P, R}\left[\mathcal{B}_{P \cup R}^{1, n}\right]$ is

$$
\mathcal{U}_{P, R}\left[\mathcal{B}_{P \cup R}^{1, n}\right]=\left\{(\pi, \rho) \in \mathrm{S}_{P} \times \mathrm{S}_{R} \mid \quad \pi \oplus \rho \in \mathcal{B}_{P \cup R}^{1, n}, \quad \underset{s_{1 P}=0}{\operatorname{Res}} A(1, \pi, \rho, n) \neq 0\right\}
$$

where $\pi$ and $\rho$ are suborderings and $\pi \oplus \rho$ is their concatenation. In order to prove the colour-factorisation formula (2.2) by equating eqs. (2.7) and (2.8), we need to be able to identify the corresponding permutations $\sigma_{1}=\pi$ and $\sigma_{2}=\rho$. For this to be true, the two sets of cut permutations must be identical. In other words, colour factorisation relies on an important property connecting bases of different multiplicities

$$
\mathcal{U}_{P, R}\left[\mathcal{B}_{P \cup R}^{1, n}\right]=\mathcal{B}_{P}^{1, \bar{p}} \times \mathcal{B}_{R}^{p, n}
$$

We dub a set of bases satisfying this constraint "co-unitary." We construct such a set of bases for all multi-quark amplitudes in section 3 .

From this derivation it is clear why the particle $p$ needs to exist: otherwise the factorisation limits yield zero and therefore no constraint. As previously mentioned, if $p$ were to always exist, then we necessarily end up with a comb structure for colour factors, which is not the case for the decomposition of ref. [24] corresponding to a $q \bar{q}$ stretch. This occurs when the total flavour quantum numbers of the set $\{1, P\}$ cannot be balanced by a single particle. Nevertheless, we can still find constraints from kinematic factorisation.

\subsection{Leg-exchange relation and colour-ordered splitting}

Let us now show that for the leg-exchange relations (2.3)-(2.5) to hold, we require a basis which respects "colour-ordered splitting". We once again privilege two legs, 1 and $n$, and now additionally pick two other particles, $i$ and $j$, in a colour-dressed amplitude. On the one hand, if we take the corresponding two-particle factorisation limit and then colour- 
decompose it, we obtain

$$
\begin{aligned}
& \operatorname{Res}_{s_{i j}=0} \mathcal{A}(\underbrace{i}_{n} \overbrace{}^{i}) \\
& =\mathcal{A}(\underbrace{P}_{n}) \mathcal{A}(\overbrace{}^{i} \gamma^{j})
\end{aligned}
$$

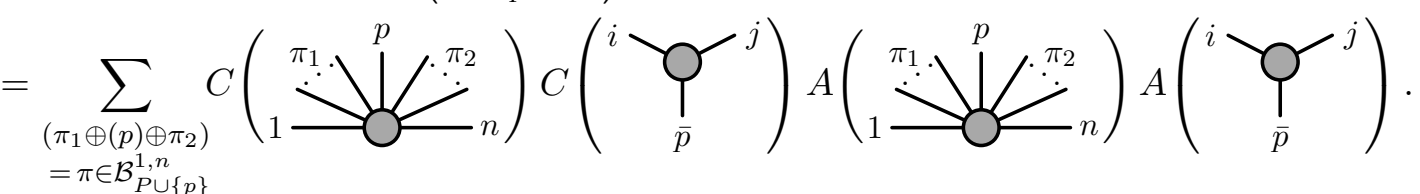

Here the set of the unselected legs $P=\{2, \ldots, n-1\} \backslash\{i, j\}$ is permuted along with the cut leg $p$, and in the resulting permutations $\pi=\pi_{1} \oplus(p) \oplus \pi_{2}$ we track the position of $p$.

On the other hand, applying the same factorisation limit to the already colourdecomposed amplitude yields

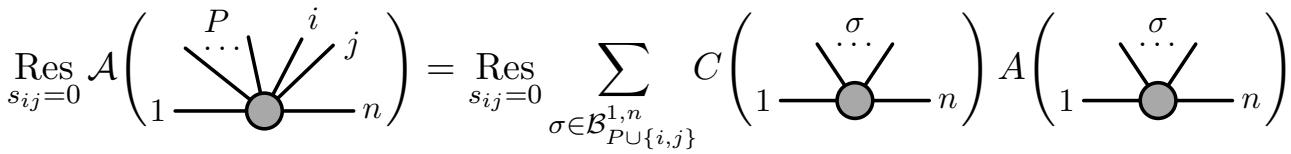

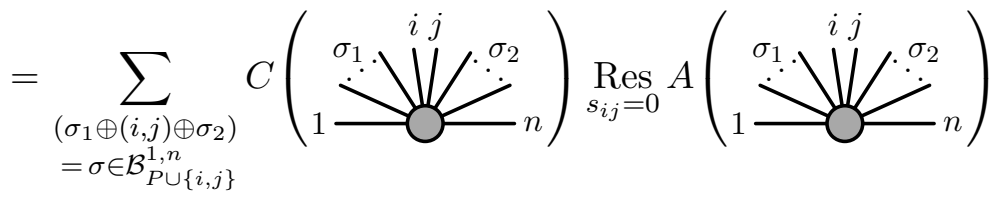

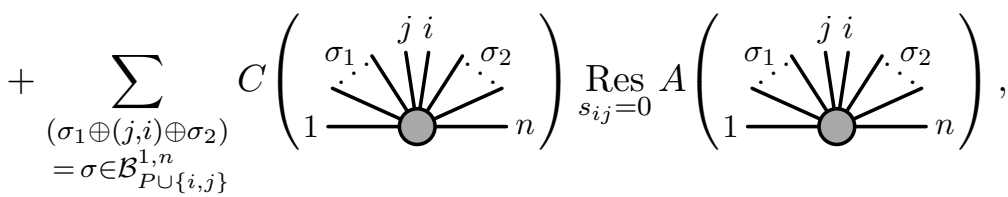

where we consider only the colour-ordered amplitudes with particles $i$ and $j$ next to each other, as only they could possibly have a non-zero residue in that limit. We note that a given colour decomposition may not actually include both terms in (2.12). Nevertheless, generically we can take this residue as

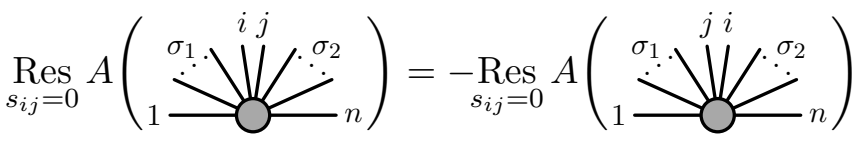

$$
\begin{aligned}
& =A(\underbrace{\sigma_{1} \cdot \underbrace{\sigma_{2}}_{n}}_{1}) A(\underbrace{i} \gamma^{j}) .
\end{aligned}
$$

Given this relation, we can rearrange equation (2.12) such that the kinematic part of the summand now takes the same form as that of (2.11). In order to equate the colour factors of these two representations, we need to be able to identify the set of permutations. That is, it must be possible to construct all terms in the $n$-point basis of colour-ordered amplitudes where $i$ and $j$ are found next to each other by taking the basis of $(n-1)$-point amplitudes 
and replacing the particle $p$ which balances all quantum numbers of the pair $(i, j)$. This operation has a physical interpretation as an ordered "splitting" of the particle $p$ into the pair $(i, j)$, and so we require the bases to behave naturally under splittings. More precisely, for any ordered pair $(i, j)$ among the unstretched particles in the $n$-point basis, we require that the bases satisfy

$$
\left.\mathcal{B}_{P \cup\{p\}}^{1, n}\right|_{p \rightarrow(i, j)}=\left\{\sigma_{1} \oplus(i, j) \oplus \sigma_{2}=\sigma \in \mathcal{B}_{P \cup\{i, j\}}^{1, n}\right\} .
$$

For a basis that satisfies this relation, the kinematic parts of eqs. (2.11) and (2.12) can be identified, allowing us to equate the permutations $\sigma_{i}$ with $\pi_{i}$. In section 3.4.2 we show that, up to the potential vanishing of terms in this derivation, the bases of colour-ordered amplitudes considered in this work indeed satisfy (2.14), leading us to the leg-exchange relations $(2.3)-(2.5)$.

\section{Co-unitary tree-level bases and colour decompositions}

In this section we discuss various colour decompositions of tree-level QCD amplitudes. Each decomposition corresponds to a basis of ordered amplitudes with two chosen particles fixed next to each other. These bases of amplitudes are independent with respect to the KK relations, i.e. over the field of rational numbers. For tree-level amplitudes with $n$ particles and $k$ quark lines, any such basis is composed from $(n-2) ! / k$ ! independent elements [27]. We leave discussion of amplitude bases with respect to the kinematically dependent BernCarrasco-Johansson [24, 34] relations for future work. Here we shall see that the colour factors associated with the KK-independent bases are given by the colour diagrams (or sets thereof) that resemble combs stretched by the fixed particles from two sides.

\subsection{Like-flavour stretch}

For completeness and in order to introduce concepts that will be important at later stages, we begin by reviewing the amplitude basis with two quarks of the same flavour fixed next to each other introduced by Melia in refs. [26, 27], as well as the corresponding colour decomposition ref. [24]. Further, we will show how this decomposition is fixed by factorisation.

$\boldsymbol{q} \overline{\boldsymbol{q}}$ amplitude basis. The basis of ordered amplitudes $A(\underline{1}, \overline{2}, \sigma),{ }^{3}$ with two like-flavoured quarks fixed is built from permutations $\sigma$ of the remaining $(n-2)$ labels. These allow gluons in arbitrary positions but impose more structure on the positions of the quark labels. A simple way to describe this is that the quark labels must correspond to legal "bracket structures". For definiteness we associate the quark labels with opening brackets " $\{$ " and

\footnotetext{
${ }^{3}$ We follow the notation of ref. [24] for labeling the external particles. Specifically, we consider all $k$ pairs of quarks and antiquarks to be of distinct flavours; for definiteness we use odd underscored labels $\underline{1}, \underline{3}, \ldots$ for quarks and even overscored labels $\overline{2}, \overline{4}$ for their respective antiquarks. The remaining $(n-2 k)$ gluons are labeled with plain numbers such as 5 . Within graphs, we find it convenient to distinguish the flavours of the quark lines by different colourings. Amplitudes with multiple quark pairs of the same flavour may be obtained from equal-mass, distinct-flavour amplitudes by summing over permutations of a chosen subset of quark labels, see eq. (4.2) below.
} 
antiquark labels with closing brackets "\}". Intuitively, a closing bracket must always follow an opened and previously unclosed bracket, so brackets structures like \{\}$\}\{\{\}$ are illegal. All such opening-closed bracket pairs correspond to quark-antiquark pairs. We can further embellish these bracket labels with colour in order to distinguish the flavour information. For instance, we can equate the permutation $(\underline{3}, \underline{5}, \overline{6}, \underline{7}, \overline{8}, \overline{4})$ to the bracket structure $\{\{\}\{\}\}$.

Naturally, all bracket structures can be seen as arising from a recursive construction from shorter bracket structures, that is

$$
k \text {-bracket }=\{(i-1) \text {-bracket }\} \oplus(k-i) \text {-bracket. }
$$

We can precisely formulate this in a recursive definition for all flavoured quark brackets. For a given set of flavours $F$, the set of associated bracket structures is given by

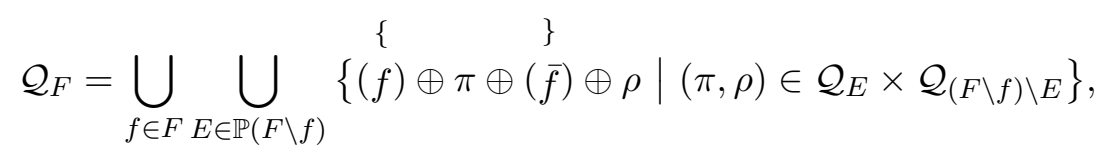

where $\mathbb{P}(S)$ is the set of all subsets of $S$, or the power set of $S$. The base of the above recursion is $\mathcal{Q}_{\emptyset}$, which contains only an empty ordering (). This formulation is useful as it will allow us to easily extend to the results of the following sections. Furthermore, it provides a way to compute how many such brackets there are:

$$
\left|\mathcal{Q}_{2 k}\right|=\sum_{i=1}^{k}\left(\begin{array}{l}
k \\
i
\end{array}\right)\left(\begin{array}{l}
i \\
1
\end{array}\right)\left|\mathcal{Q}_{2(i-1)}\right|\left|\mathcal{Q}_{2(k-i)}\right|=\frac{(2 k) !}{(k+1) !}
$$

where $\mathcal{Q}_{2 k}$ is a shorthand for the set of distinctly flavoured quark brackets of length $2 k$.

The above notation allows us to formulate Melia's amplitude basis as ${ }^{4}$

$$
\mathcal{B}_{n, k}^{2,1}=\left\{A(\overline{2}, \sigma, \underline{1}) \mid \sigma \in \mathcal{Q}_{2(k-1)} \amalg \mathcal{G}_{n-2 k}\right\},
$$

where the unfixed quark brackets in $\mathcal{Q}_{2(k-1)}$ are shuffled ${ }^{5}$ with arbitrary permutations of gluon labels, as denoted by

$$
\mathcal{G}_{n-2 k}=\left\{\sigma \in \mathrm{S}_{G} \mid G=\left\{g_{2 k+1}, \ldots, g_{n}\right\}\right\} .
$$

The size of the basis is then immediately [27]

$$
\left|\mathcal{B}_{n, k}^{1,2}\right|=\left|\mathcal{Q}_{2(k-1)}\right| \times(n-2 k) ! \times \frac{(n-2) !}{(2 k-2) !(n-2 k) !}=\frac{(n-2) !}{k !} .
$$

Recall that the cyclic symmetry of colour-ordered amplitudes allows us to freely rewrite $A(\overline{2}, \sigma, \underline{1})$ as $A(\underline{1}, \overline{2}, \sigma)$. For the five-point four-quark amplitude considered in section 1.1

\footnotetext{
${ }^{4}$ In contrast to the notation used for the the basis set of permutations associated to a basis, here we use a similar notation for the basis set of ordered amplitudes, $\mathcal{B}_{n, k}^{a, b}=\left\{A(a, \chi, b) \mid \chi \in \mathcal{B}_{X}^{a, b}\right\}$, highlighting the particle and quark-pair counts $n$ and $k$.

${ }^{5}$ The standard definition of the shuffle product $\rho ш \sigma$ of two orderings of length $r$ and $s$ is the set of $(r+s) ! /(r ! s !)$ ways of interleaving them, i.e. permuting $\rho \oplus \sigma$ without changing their internal order.
} 
only the quark pair $\underline{3} \leftarrow \overline{4}$ is unfixed, so the single bracket structure \{\} is dressed with three gluon insertions and gives the Melia basis of $A(\underline{1}, \overline{2}, 5, \underline{3}, \overline{4}), A(\underline{1}, \overline{2}, \underline{3}, 5, \overline{4})$ and $A(\underline{1}, \overline{2}, \underline{3}, \overline{4}, 5)$.

It is worth pointing out that the quark-arrow convention for each quark pair may be switched at will. So the Melia basis $\{A(\underline{1}, \sigma, \overline{2})\}$ (used e.g. in ref. [35]) is equivalent to $\{A(\overline{2}, \sigma, \underline{1})\}$, as well as their relabelings $\{A(\underline{2}, \sigma, \overline{1})\}$ and $\{A(\overline{1}, \sigma, \underline{2})\}$. In the specific rendition of the Melia basis above the arrow of the base quark line $\overline{2} \rightarrow \underline{1}$ goes in the opposite way to the rest. This can be depicted by an outer pair of square brackets. For instance, the above five-point orderings are $(\overline{2}, 5, \underline{3}, \overline{4}, \underline{1})=[5\{\}],(\overline{2}, \underline{3}, 5, \overline{4}, \underline{1})=[\{5\}]$ and $(\overline{2}, \underline{3}, \overline{4}, 5, \underline{1})=[\{\} 5]$. Square brackets will play a more important role in the subsequent sections, while at this point they are a matter of convention for the fixed quark pair.

$\boldsymbol{q} \overline{\boldsymbol{q}}$ colour decomposition. In order to rewrite the full colour-dressed amplitude $\mathcal{A}_{n, k}$ in terms of the Melia basis of ordered amplitudes, we need to characterise the corresponding colour coefficients $C(\underline{1}, \overline{2}, \sigma)$ in the decomposition

$$
\mathcal{A}(\underline{1}, \overline{2}, X)=\sum_{\sigma \in \mathcal{B}_{X}^{1,2}} C(\underline{1}, \overline{2}, \sigma) A(\underline{1}, \overline{2}, \sigma) .
$$

They are given by certain vertical-ladder colour diagrams most easily understood graphically [24]. For instance, for the $q \bar{q}$ stretch of the five-point amplitude studied in section 1.1 this decomposition involves the following colour factors:

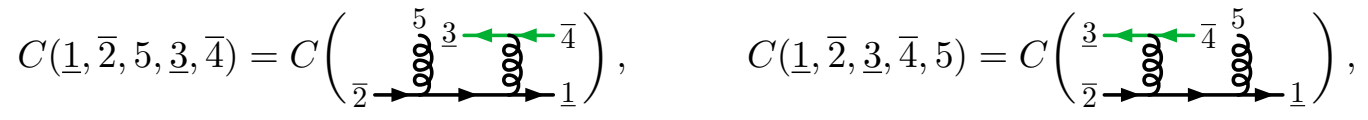

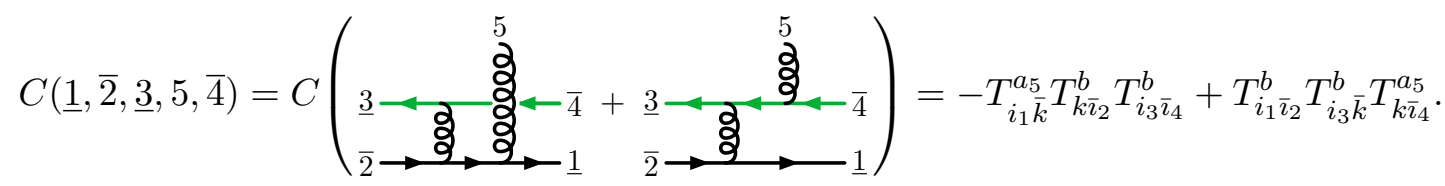

Here the last line contains more than one diagram due to gluon 5 being in a nested position $[\{5\}]$ - surrounded by more than one pair of quark brackets. Moreover, the first two colour coefficients illustrate the fact that the quark pair $\underline{3} \leftarrow \overline{4}$ as a whole behaves much like a gluon, so it easy to imagine that a quark pair in a nested position with respect to other quarks also generates summation over different ways to contract its adjoint index (shown by the curly line).

A general construction for the colour coefficients in eq. (3.7) can be implemented by the following algebraic formula

$$
C(\underline{1}, \overline{2}, \sigma)=(-1)^{k-1}[2|\sigma| 1] \mid \begin{aligned}
g & \rightarrow \Xi_{l(g)}^{a_{g}} \\
q & \rightarrow\left\{q \mid T^{b} \otimes \Xi_{l(q)-1}^{b}\right. \\
\bar{q} & \rightarrow \mid q\}
\end{aligned}
$$

where a bra-ket notation represents the fundamental indices, e.g.

$$
\left\{q\left|T^{a}\right| \bar{q}\right\}=T_{i_{q} \bar{\imath}_{\bar{q}}}^{a}, \quad\left[2\left|T^{a}\right| 1\right]=T_{\bar{\imath}_{2} i_{1}}^{a}=-T_{i_{1} \bar{\tau}_{2}}^{a} .
$$


Then the replacement rules in eq. (3.9) convert a bracketed permutation $\sigma$ to an expression in terms of the usual generators $T^{a}$ and new tensor-representation generators

$$
\begin{aligned}
& \Xi_{l}^{a}=\sum_{r=1}^{l} \underbrace{\mathbb{I} \otimes \cdots \otimes \mathbb{I} \otimes \overbrace{T_{r}^{a} \otimes \mathbb{I} \otimes \cdots \otimes \mathbb{I} \otimes \overline{\mathbb{I}}}^{r} .}_{l}
\end{aligned}
$$

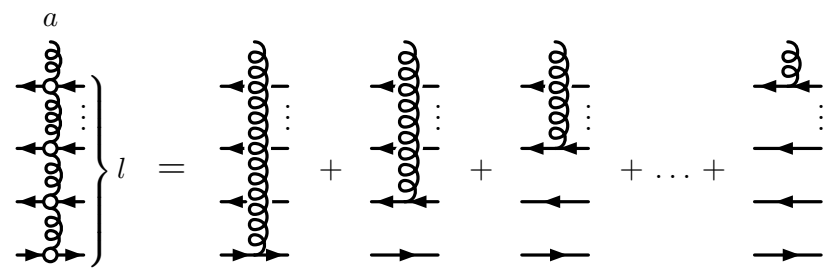

They have an explicit dependence on the nestedness level $l$ of the label being replaced within eq. (3.9) and encode the summation over different ways to attach an adjoint index to the quark lines of the surrounding brackets. ${ }^{6}$ The closed-form construction above was conjectured by Johansson and one of the current authors [24] and subsequently proven by Melia [25]. Whenever we wish to invoke it, we will refer to the colour factors (3.9) as $C_{\mathrm{JO}}(\overline{2}, \sigma, \underline{1})$. Moreover, as any like-flavoured quarks can be exchanged, the colour factors

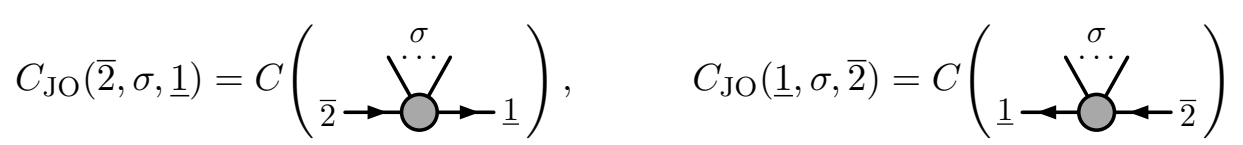

differ from each other by a trivial flip of the baseline arrow in eq. (3.11).

It is worth noting that although the colour factors are uniquely specified by the Melia basis (3.4), they can be expressed in different ways, and the above formulae give one of many possible colour-diagrammatic representations for them. For instance, the five-point colour factor $C(\underline{1}, \overline{2}, \underline{3}, 5, \overline{4})$ is shown in eq. (3.8) as a combination of two diagrams with the adjoint line of gluon 5 on the right-hand side of the quark line $\underline{3} \leftarrow \overline{4}$, but can also be rewritten with the gluonic line on the left-hand side.

A remarkable and previously unnoticed property of the JO colour factors is that they indeed satisfy the colour factorisation relation (2.2) and the leg-exchange relations (2.3)(2.5). These properties indeed follow from the co-unitarity of the basis to be shown in section 3.4.1. Alternatively, they can be derived from the definition of the colour factors (3.9) and the algebraic relations (2.6), as detailed in appendix B.

$\boldsymbol{q} \overline{\boldsymbol{q}}$ colour from factorisation. Let us now present an argument showing that the counitarity of the Melia basis to itself also completely fixes the JO colour factors in its associated decomposition. In short, one can systematically apply either the colour factorisation relation or the leg-exchange relation until the colour factor is entirely expressed in terms of three-point colour vertices. Such a procedure is not unique, reflecting the non-uniqueness

\footnotetext{
${ }^{6}$ As an explicit example, we apply the formula (3.9) to the non-trivial five-point colour factor in eq. (3.8):

$$
\begin{aligned}
C(\underline{1}, \overline{2}, \underline{3}, 5, \overline{4}) & =C[2,\{3,5,4\}, 1]=-\left[2\left|\left\{3\left|T^{b} \otimes \Xi_{1}^{b}\right| \Xi_{2}^{a_{5}} \mid 4\right\}\right| 1\right]=-\left[2\left|\left\{3\left|T^{b} \otimes T^{b}\right| T^{a_{5}} \oplus T^{a_{5}} \mid 4\right\}\right| 1\right] \\
& =-\left[2\left|T^{b} T^{a_{5}}\left\{3\left|T^{b}\right| 4\right\}\right| 1\right]-\left[2\left|T^{b}\left\{3\left|T^{b} T^{a_{5}}\right| 4\right\}\right| 1\right]=-T_{\bar{\imath}_{2} k}^{b} T_{\bar{k} i_{1}}^{a_{5}} T_{i_{3} \bar{\imath}_{4}}^{b}-T_{\bar{\imath}_{2} i_{1}}^{b} T_{i_{3} \bar{k}}^{b} T_{k \bar{\imath}_{4}}^{a_{5}} .
\end{aligned}
$$
}


of the closed-form representation for the colour factors. Nevertheless, it provides a useful recursive way of implementing these colour factors, as the only place where any convention may enter is in the definition of the three-point colour factors.

We will make use of three identities. The first two are the colour factorisation relation (2.2) and the leg-exchange relation for a $q \bar{q}$ pair (2.3). As all amplitudes in the Melia basis have a specific orientation of quark brackets, both of these equations relate $n$-point colour factors to $(n-1)$-point ones. The third relation we shall employ is the quark-gluon exchange relation (2.4), which necessarily involves two terms of the same multiplicity. For this reason it can be regarded as the origin of the multiple terms in the JO colour factors.

Our aim is to apply the three relations in such a way that we can always decrease the multiplicity of the involved diagrams. This will necessarily result in a multi-parton colour factor expressed in terms of three-point colour vertices. We consider each identity in turn.

1. We can apply the colour factorisation relation (2.2) unless the first and last particles of the permutation are a quark and antiquark of the same flavour, where flavour conservation makes this impossible. If it is possible, we apply the factorisation identity and thus reduce the multiplicity of the involved diagrams.

2. Otherwise we look to apply the leg-exchange relation (2.3) for a $q \bar{q}$ pair. This is possible if there is an adjacent $q \bar{q}$ pair in the permutation, in which case we immediately reduce the multiplicity of the involved diagrams.

3. If we cannot find such a pair, then there must be one or more gluons enclosed by the most nested quark bracket. We can then repeatedly use the $q g$ exchange relation (2.4) to move these gluons to the right of all the quarks in the permutation. This introduces a number of additional terms, but they involve diagrams of lower of multiplicity. The remaining terms of the same multiplicity no longer have all particles enclosed and are therefore again amenable to the colour factorisation identity.

In this way, we can repeatedly apply the three relations until we reach an expression for the colour factor entirely given in terms of three-point vertices. Notably, we chose to move all gluons to the right, which corresponds to the non-uniqueness of the JO factors.

To demonstrate the above factorisation procedure, let us derive the five-point colour factor presented earlier in eq. (3.8b):

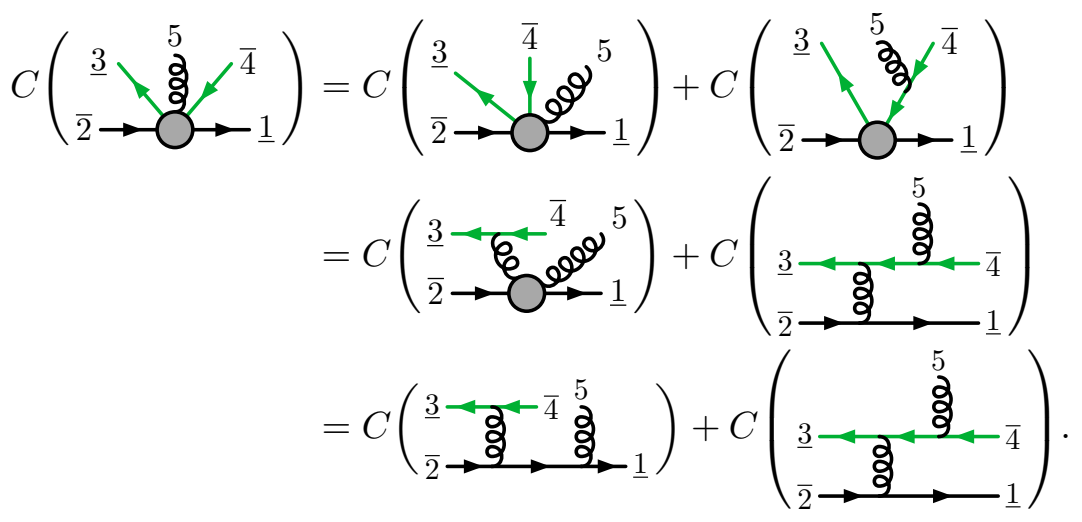


Here the starting diagram cannot be directly factorised via the relations (2.2) or (2.3), so in the first line we move gluon 5 using the quark-gluon exchange relation (2.4). The resulting additional term contains a three-point vertex connected to a four-point amplitude subdiagram, ${ }^{7}$ which can then be converted to an explicit colour factor via the $q \bar{q}$ factorisation identity (2.3). This identity also factorises the remaining five-point term onto a four-point sub-diagram, which is finally amenable to the basic colour factorisation relation (2.2).

\subsection{Distinct-flavour stretch}

Starting from this section, we introduce new bases of colour-ordered amplitudes in QCD, as well as the corresponding colour decompositions. We leave a detailed discussion of their properties to section 3.4.1.

$q Q$ amplitude basis. We begin with the case of two distinctly flavoured quarks fixed next to each other. Without loss of generality, let us label the fixed particles as quark 1 and antiquark $\overline{4}$. Within the basis of ordered amplitudes, any ordering then starts with a standard opening bracket "\{" and ends with a standard closing bracket " \}". The consistency of the bracket structure implies that the former must be closed with "\}" $\Leftrightarrow \overline{2}$ before the latter is opened with " $\{" \Leftrightarrow \underline{3}$. So in contrast to the $q \bar{q}$ case, the particles appearing in-between are not automatically enclosed by any external brackets. Now in order to build a set of permutations that corresponds to an amplitude basis, one needs to introduce a new feature to our bracket structures - all unenclosed quark brackets should be allowed to appear in both kinds of arrow orientations. More enclosed quark pairs, however, should only come with one of two orderings, which we here choose to be the canonical one. We note that this is indeed a choice of a linearly independent set of amplitudes, corresponding to a given "signature" of quark line orientation [27]. For instance, brackets structures like \{\}$\{\{\}\}\{\},\{\}[\{\}]\{\},\{\}[][]\{\}$ or $\{\{\}\}[]\}\}$ are now all legal, but \{\}$\{[]\}\{\},\{\}[[]]\{\}$ or $\{[]\}[]\{\}$ are not. This pattern is consistent with the observations of ref. [33] at one loop. Note that this feature does not arise until six-point amplitudes, as a $q Q$ basis for the fivepoint amplitude considered in section 1.1 can be shown to be $\{5\}\{\},\{\} 5\{\}$ and \{\}$\{5\}$. To be more illustrative of the new features of the basis, let us present a six-quark basis in the context of a complete colour decomposition

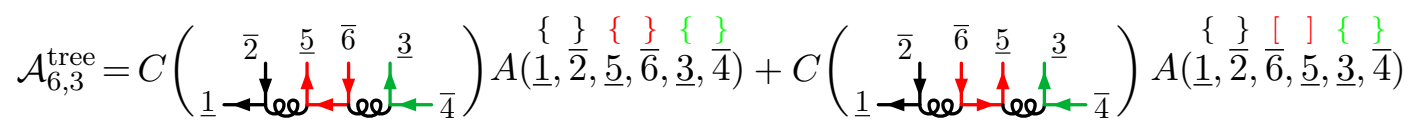

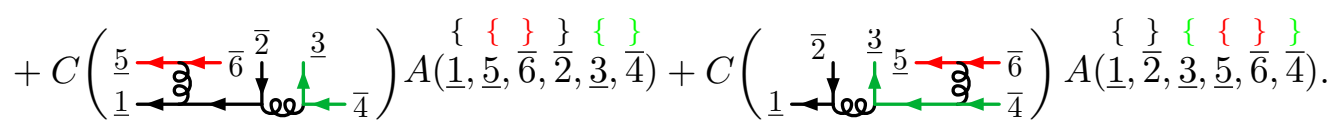

The form of the colour factors here can be derived from the principal unitarity constraint (2.2) and the five-point colour factors (1.5). Unlike in Melia's basis, the $q Q$-stretched base line is now separated into multiple segments, the leftmost and rightmost being $\underline{1} \leftarrow \overline{2}$ and $\underline{3} \leftarrow \overline{4}$ with possible other particles in-between.

\footnotetext{
${ }^{7}$ In eq. (3.14) and below we use a slightly streamlined notation with respect to e.g. eqs. (2.2)-(2.5) by allowing both amplitude blob diagrams and explicit vertices within a single colour-factor diagram.
} 
Intuitively, all $k$-bracket structures with both orientations of unenclosed brackets can be divided into its first such bracket with only unflipped brackets inside and a similar remainder,

$$
k \text {-bracket w/ flips }=\left\{\begin{aligned}
& \text { either }\{(i-1) \text {-bracket }\} \oplus(k-i) \text {-bracket w/ flips } \\
& \text { or }[(i-1) \text {-bracket }] \oplus(k-i) \text {-bracket w/ flips }
\end{aligned}\right.
$$

We can precisely capture this behaviour by introducing a set of all quark brackets with flips allowed for those in an unenclosed position:

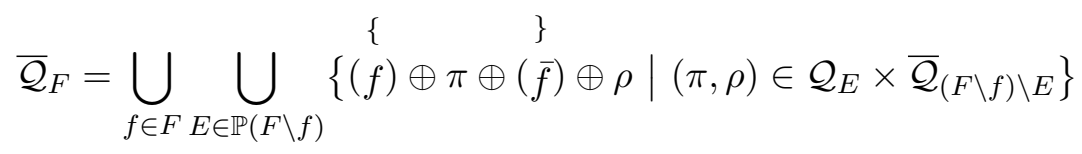

$$
\begin{aligned}
& \cup\left\{(\bar{f}) \oplus \pi \oplus(f) \oplus \rho \mid(\pi, \rho) \in \mathcal{Q}_{E} \times \overline{\mathcal{Q}}_{(F \backslash f) \backslash E}\right\},
\end{aligned}
$$

where each time an enclosed bracket occurs, it is taken from $\mathcal{Q}_{F}$ defined previously in eq. (3.2). Again, the base of the recursion is that $\overline{\mathcal{Q}}_{\emptyset}$ is a set with an empty ordering. The recursive nature of this definition allows us to easily obtain a counting for such structures:

$$
\left|\overline{\mathcal{Q}}_{2 k}\right|=\sum_{i=1}^{k} 2\left(\begin{array}{l}
k \\
i
\end{array}\right)\left(\begin{array}{l}
i \\
1
\end{array}\right)\left|\mathcal{Q}_{2(i-1)}\right|\left|\overline{\mathcal{Q}}_{2(k-i)}\right|=\frac{(2 k) !}{k !}
$$

where the factor of two accounts for the canonical and flipped bracket configurations of the first bracket pair comprising $(i-1)$ standard brackets and followed by the remaining bracket structures with flips.

In terms of the above set, we define the new basis as

$$
\mathcal{B}_{n, k}^{1,4}=\left\{A(\underline{1}, \sigma, \overline{4}) \mid(\underline{1}) \oplus \sigma \oplus(\overline{4}) \in \overline{\mathcal{Q}}_{2 k} \amalg \mathcal{G}_{n-2 k}\right\}
$$

where $k>2$ and the concatenation $(\underline{1}) \oplus \sigma \oplus(\overline{4})$ explicitly selects only those elements of $\overline{\mathcal{Q}}_{2 k}$ that begin with $\underline{1}$ and end with $\overline{4}$. We note that whilst the set is too large to describe the present basis, this is because it unifies the treatment of all bases described in this paper. Let us now check that for the basis (3.19) its size equals that of the Melia basis (3.4). For that, we construct a pure-quark bracket as

$$
\{(i-1) \text {-bracket }\} \oplus(j-i) \text {-bracket w/ flips } \oplus\{(k-j-1) \text {-bracket }\} \text {. }
$$

Its counting is then computed as

$$
\left|\mathcal{B}_{n=2 k, k}^{1,4}\right|=\sum_{i=1}^{k-1} \sum_{j=i}^{k-1}\left(\begin{array}{c}
k-2 \\
i-1
\end{array}\right)\left(\begin{array}{l}
k-i-1 \\
k-j-1
\end{array}\right)\left|\mathcal{Q}_{2(i-1)}\right|\left|\overline{\mathcal{Q}}_{2(j-i)}\right|\left|\mathcal{Q}_{2(k-j-1)}\right|=\frac{(2 k-2) !}{k !}
$$

This is indeed $\left|\mathcal{Q}_{2(k-1)}\right|$, so dressing the quark bracket structures with gluons promotes it to the required $(n-2) ! / k$ ! analogously to eq. (3.6). 
$q Q$ colour decomposition. Let us discuss the colour factors in the decomposition onto the distinct-flavour basis

$$
\mathcal{A}(\underline{1}, X, \overline{4})=\sum_{\sigma \in \mathcal{B}_{X}^{1,4}} C(\underline{1}, \sigma, \overline{4}) A(\underline{1}, \sigma, \overline{4}) .
$$

In order to formulate a closed-form expression for the colour factors, we note that any given permutation $\sigma$ is naturally split by the quarks in the unnested positions into $(2 u-1)$ sub-permutations, e.g.

$$
(\underline{1}, \sigma, \overline{4})=\left(\left\{1, \sigma_{1}, 2\right\}, \sigma_{2},\left\{5, \sigma_{3}, 6\right\}, \sigma_{4}, \ldots, \sigma_{2 u-2},\left\{3, \sigma_{2 u-1}, 4\right\}\right), \quad 2 \leq u \leq k,
$$

where the evenly numbered $\sigma_{2 v}$ are defined to be purely gluonic. Since all permutations in the $q Q$ basis (3.19) can be obtained from this configuration by relabelling, writing its colour factor is sufficient to specify the complete decomposition (3.22). We can now use this breakdown of the permutation to find a closed form for the colour factor in terms of previously defined building blocks. By construction, the colour factorisation formula (2.2) allows us to write

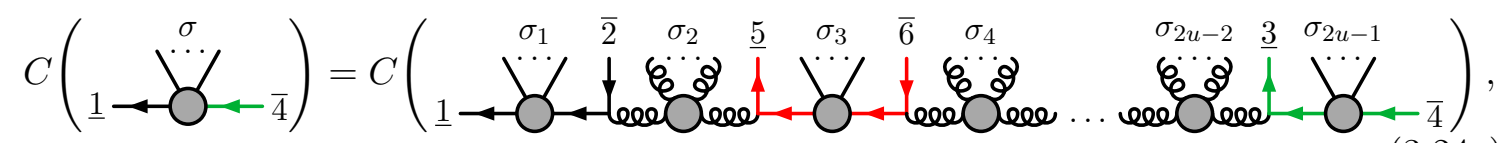

which translates to

$$
\begin{aligned}
C(\underline{1}, \sigma, \overline{4})_{\imath_{1} \bar{\imath}_{4}}= & C_{\mathrm{JO}}\left(\underline{1}, \sigma_{1}, \overline{2}\right)_{\imath_{1} \bar{\jmath}_{2}} T_{\jmath_{2} \bar{\imath}_{2}}^{b_{1}} C_{\mathrm{DDM}}\left(g_{1}, \sigma_{2}, g_{2}\right)^{b_{1} b_{2}} \\
& \times \prod_{v=3}^{u}\left\{T_{\imath_{2 v-1} \bar{\jmath}_{2 v-1}}^{b_{2 v-4}} C_{\mathrm{JO}}\left(\underline{2 v-1}, \sigma_{2 v-3}, \overline{2 v}\right)_{\jmath_{2 v-1} \bar{\jmath}_{2 v}} T_{\jmath_{2 v} \bar{\imath}_{2 v}}^{b_{2 v-3}}\right. \\
& \left.\times C_{\mathrm{DDM}}\left(g_{2 v-3}, \sigma_{2 v-2}, g_{2 v-2}\right)^{b_{2 v-3} b_{2 v-2}}\right\} T_{\imath_{3} \bar{\jmath}_{3}}^{b_{2 u-2}} C_{\mathrm{JO}}\left(\underline{3}, \sigma_{2 u-1}, \overline{4}\right)_{\jmath_{3} \bar{\tau}_{4}} .
\end{aligned}
$$

Here we have only made explicit the colour indices relevant for the factorisation. Recall that the $q \bar{q}$-stretch colour factors have been introduced in eq. (3.9), whereas the DDM colour factors are given by strings of structure constants, namely

$$
C_{\mathrm{DDM}}(1, \sigma, n)=C\left(\begin{array}{c}
\sigma \\
\dot{\xi} \boldsymbol{q} \\
1 \text { eee Oees } n
\end{array}\right)=\tilde{f}^{a_{1} a_{\sigma(2)} b_{1}} \tilde{f}^{b_{1} a_{\sigma(3)} b_{2}} \ldots \tilde{f}^{b_{n-3} a_{\sigma(n-1)} a_{n}} .
$$

We supplement it with the trivial extension $C_{\mathrm{DDM}}(1,2)^{a_{1} a_{2}}=\delta^{a_{1} a_{2}}$ to account for the case where a purely gluonic permutation $\sigma_{2 v}$ in (3.24) happens to be empty.

As an amusing switch of perspective, one could now regard the $q \bar{q}$ colour factors (3.9) as a particular case of the above $q Q$ colour factors, for which no initial factorisation into flavour-neutral blocks, as in eq. (3.24a), is possible.

\section{3 $\quad$ Stretching with gluons}

In this section we present the amplitude bases and the corresponding colour decompositions for the cases where one or both fixed particles are gluons. 
Quark-gluon stretch. Without loss of generality, we take the fixed quark and gluon to be $\underline{1}$ and $n$. Then the new basis is defined as

$$
\mathcal{B}_{n, k}^{1, n}=\left\{A(\underline{1}, \sigma, n) \mid(\underline{1}) \oplus \sigma \in \overline{\mathcal{Q}}_{2 k} ш \mathcal{G}_{n-2 k}\right\}
$$

where $n>2 k$. Similarly to the $q Q$ basis (3.19), the quark lines that appear in an unnested position to right of label $\overline{2}$ may come in two orientations. Conveniently, we have already considered a five-point example of such a basis in section 1.1, and in the bracket notation it is given by \{\}\{\} $5,\{\}[] 5$ and $\{\{\}\} 5$. As the basis is constructed from the same set of quark brackets as the $q Q$ basis, the same rules about nesting of square brackets apply. For example, if we consider a seven-point six-quark basis this involves orderings corresponding to \{\}$[\{\}] 7$ and \{\}[][] 7 , but not \{\}$[[]] 7$.

There are multiple ways to define the amplitude decomposition into the $q g$ basis,

$$
\mathcal{A}(\underline{1}, X, n)=\sum_{\sigma \in \mathcal{B}_{X}^{1, n}} C(\underline{1}, \sigma, n) A(\underline{1}, \sigma, n) .
$$

Here we choose to define it by factorising onto the $q Q$ colour factors (3.24). In other words, since every permutation $\sigma$ may be split into two by the rightmost-occurring quark, which we denote as $\bar{q}$, we depict the $q g$ colour factors as

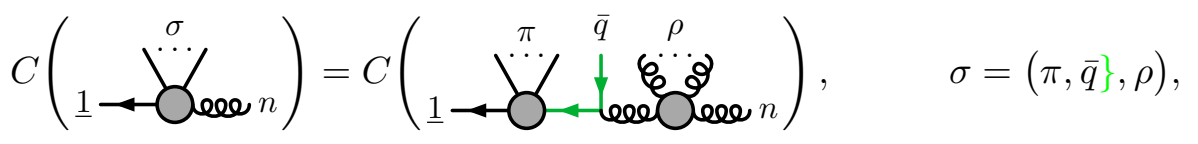

More explicitly, we write

$$
C(\underline{1}, \sigma, n)_{\imath_{1}}^{a_{n}}=C(\underline{1}, \pi, \bar{q})_{\imath_{1} \bar{\jmath}}\left[-T_{j \bar{\jmath}_{\bar{q}}}^{b}\right] C_{\mathrm{DDM}}(g, \rho, n)^{b a_{n}} .
$$

In the particular case where the rightmost quark $\bar{q}$ is $\overline{2}$, the distinct-flavour colour factor $C(\underline{1}, \pi, \bar{q})$ should be replaced by the like-flavour one (3.9), which can otherwise be considered as its degeneration.

Gluon-gluon stretch. Now fixing two gluons next to each other and labelling them as $(n-1)$ and $(n-2)$, we define a new basis and the corresponding colour decomposition as

$$
\begin{aligned}
\mathcal{B}_{n, k}^{n-1, n} & =\left\{A(n-1, \sigma, n) \mid \sigma \in \overline{\mathcal{Q}}_{2 k} ш \mathcal{G}_{n-2 k-2}\right\}, \\
\mathcal{A}(n-1, X, n) & =\sum_{\sigma \in \mathcal{B}_{X}^{n-1, n}} C(n-1, \sigma, n) A(n-1, \sigma, n),
\end{aligned}
$$

where $n \geq 2 k+2$. Since no quark label is fixed, we should now allow all unenclosed quark brackets to appear in both orientations, while brackets in nested positions are chosen in the standard orientation. For example, a six-parton amplitude with two gluons can be expanded in the basis of

$$
\begin{array}{llll}
5\{\}\{\} 6, & 5\{\}[] 6, & 5[]\{\} 6, & 5[][] 6, \\
5\{\}\{\} 6, & 5\{\}[] 6, & 5[]\{\} 6, & 5[][] 6, \\
5\{\{\}\} 6, & 5[\{\}] 6, & 5\{\{\}\} 6, & 5[\{\}] 6 .
\end{array}
$$


There are many ways to formulate the $g g$-stretch colour decomposition. For instance, finding the leftmost-occuring quark $q$, we can split a DDM building block off the $q g$-stretch colour factor defined in eq. (3.28) above:

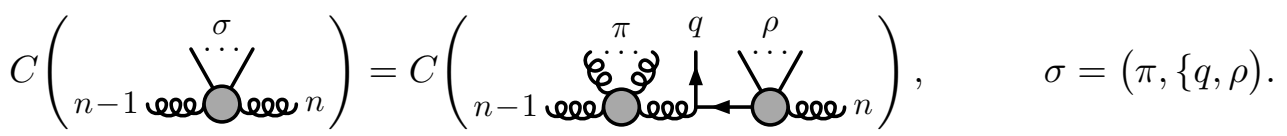

In other words, we have

$$
C(n-1, \sigma, n)^{a_{n-1} a_{n}}=C_{\mathrm{DDM}}(n-1, \pi, g)^{a_{n-1} b}\left[-T_{\imath_{\bar{j}}}^{b}\right] C(\underline{1}, \rho, n)_{\jmath}{ }^{a_{n}} .
$$

\subsection{Properties of tree-level bases}

In this section we demonstrate the crucial properties of the new amplitude bases, on which the validity and mutual consistency of their colour decompositions rely.

\subsubsection{Co-unitarity}

Here we use the definitions of the bases above to show that they satisfy the co-unitarity property (2.10) in the factorisation channel of the form $s_{1 P}=s_{n R} \rightarrow 0$, where $P$ and $R$ are non-empty particles sets covering the unfixed labels, $P \cup R=\{2, \ldots, n-1\}$. Co-unitarity of the bases is crucial for the subsequent proof of their validity as bases, as well as the ensuing colour factorisation formulae, from which any colour decomposition can be derived.

There are two special amplitude bases that in all limits of the form $s_{1 P} \rightarrow 0$ factorise onto themselves, i.e. their lower-point versions. These are the KK basis [23] in the purely gluonic case, and the Melia basis [26, 27] in presence of quarks.

Purely gluonic case. In our notation, the KK basis is

$$
\mathcal{B}_{n, 0}^{1, n}=\left\{A(1, \sigma, n) \mid \sigma \in \mathcal{B}_{P \cup R}^{1, n}\right\}, \quad \mathcal{B}_{P \cup R}^{1, n}=\mathrm{S}_{P \cup R} .
$$

In the purely gluonic case there is a non-vanishing residue $\operatorname{Res}_{s_{1 P}=0} A(1, \pi, \rho, n)$ for any two suborderings $\pi \in \mathrm{S}_{P}$ and $\rho \in \mathrm{S}_{R}$. Therefore, the definition (2.9) implies that the set of factorised suborderings is

$$
\mathcal{U}_{P, R}\left[\mathcal{B}_{P \cup R}^{1, n}\right]=\left\{(\pi, \rho) \in \mathrm{S}_{P} \times \mathrm{S}_{R}\right\}=\mathcal{B}_{P}^{1, \bar{p}} \times \mathcal{B}_{R}^{p, n},
$$

so the co-unitarity property is manifest.

Melia-basis case. Now let us consider when the factorisation limit $s_{q P}=s_{\bar{q} R} \rightarrow 0$ of an ordered amplitude in the Melia basis (3.4) gives a non-zero residue. Since quarks $q$ and $\bar{q}$ are fixed to be on different sides of the limit, flavour conservation demands that for there to be a non-zero limit, the factorisation channel must be of this flavour and therefore that no other quark may enter $P$ unless its antiquark is also in $P$. In terms of bracket structures, this means that factorisation channels of the form

$$
\operatorname{Res}_{s_{q P}=0} A\left([\underbrace{\ldots\{\ldots}_{\pi \in \mathrm{S}_{P}} \mid \underbrace{\ldots\} \ldots}_{\rho \in \mathrm{S}_{R}}]\right)=0
$$


(a)

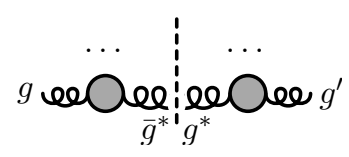

(b)

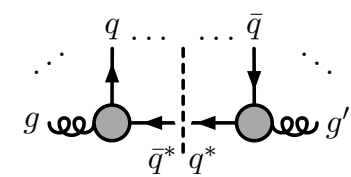

(c)

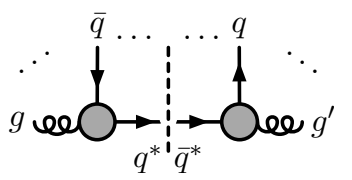

Figure 1. Factorisation channels of $g g$-basis colour-ordered amplitude.

must vanish, as opposed to

$$
\operatorname{Res}_{s_{q P}=0} A\left([\underbrace{\ldots\{\ldots\} \ldots}_{\pi \in \mathrm{S}_{P}} \mid \underbrace{\ldots\{\ldots\} \ldots]}_{\rho \in \mathrm{S}_{R}}]\right)=i A([\ldots\{\ldots\} \ldots]) A([\ldots\{\ldots\} \ldots]) \neq 0,
$$

where we have indicated the left-right separation between $P$ and $R$ by a vertical line. Therefore, denoting the quark flavours entirely in $P$ by $F_{P}$ and those in $R$ by $F_{R}$, we see no overlap between these two sets. The requisite bracket structures are $\mathcal{Q}\left(F_{P}\right)$ and $\mathcal{Q}\left(F_{R}\right)$. As $\mathcal{Q}(F)$ contains all bracket structures involving all flavours, those that survive the limit must necessarily be built from $\mathcal{Q}\left(F_{P}\right)$ and $\mathcal{Q}\left(F_{R}\right)$. Using a similar notation for the gluonic sets, $G_{P} \cup G_{R}=G=\left\{g_{2 k+1}, \ldots, g_{n}\right\}$, we can rewrite the relevant Melia bases as

$$
\mathcal{B}_{P \cup R}^{q, \bar{q}}=\mathcal{Q}\left(F_{P} \cup F_{R}\right) \amalg S_{G_{P} \cup G_{R}}, \quad \mathcal{B}_{P}^{q,,^{*}}=\mathcal{Q}\left(F_{P}\right) ш S_{G_{P}}, \quad \mathcal{B}_{R}^{q^{*}, \bar{q}}=\mathcal{Q}\left(F_{R}\right) \amalg S_{G_{R}},
$$

for which from the definition (2.9) we obtain

$$
\begin{aligned}
\mathcal{U}_{P, R}\left[\mathcal{B}_{P \cup R}^{q, \bar{q}}\right] & =\left\{(\pi, \rho) \in \mathrm{S}_{P} \times \mathrm{S}_{R} \mid \pi \oplus \rho \in \mathcal{Q}\left(F_{P} \cup F_{R}\right) \amalg S_{G_{P} \cup G_{R}}, \operatorname{Res}_{s_{q P}=0} A(q, \pi, \rho, \bar{q}) \neq 0\right\} \\
& =\left\{(\pi, \rho) \mid \pi \in \mathcal{Q}\left(F_{P}\right) \amalg S_{G_{P}}, \quad \rho \in \mathcal{Q}\left(F_{R}\right) \amalg S_{G_{R}}\right\}=\mathcal{B}_{P}^{q, \bar{q}^{*}} \times \mathcal{B}_{R}^{q^{*}, \bar{q}} .
\end{aligned}
$$

New cases. There is a significant difference between three amplitude bases proposed in this paper and the Kleiss-Kuijf and Melia bases discussed above. Namely, factorisation limits of the form $s_{1 P} \rightarrow 0$ intertwine the new bases with one another, as well as with the KK and Melia ones. In this way, the latter two are the basic cases - for this reason we considered them first. The co-unitarity property of one of the new bases depends on all of them being co-unitary at the same time.

First, we rewrite the bases as

$$
\begin{aligned}
& \mathcal{B}_{P \cup R}^{q, \bar{Q}}=\left\{\sigma \in \mathrm{S}_{P \cup R} \mid(q) \oplus \sigma \oplus(\bar{Q}) \in \overline{\mathcal{Q}}\left(F_{P} \cup F_{R}\right) \amalg S_{G_{P} \cup G_{R}}\right\}, \\
& \mathcal{B}_{P \cup R}^{q, g}=\left\{\sigma \in \mathrm{S}_{P \cup R} \mid(q) \oplus \sigma \in \overline{\mathcal{Q}}\left(F_{P} \cup F_{R}\right) \amalg S_{G_{P} \cup G_{R}}\right\}, \\
& \mathcal{B}_{P \cup R}^{g, g^{\prime}}=\overline{\mathcal{Q}}\left(F_{P} \cup F_{R}\right) \amalg S_{G_{P} \cup G_{R}},
\end{aligned}
$$

where $\overline{\mathcal{Q}}(F)$ allows both bracket orientations given that they are not enclosed. For concreteness, let us start with that corresponding to a $g g$ stretch in presence of quarks. We wish to evaluate

$$
\mathcal{U}_{P, R}\left[\mathcal{B}_{P \cup R}^{g, g^{\prime}}\right]=\left\{(\pi, \rho) \in \mathrm{S}_{P} \times \mathrm{S}_{R} \mid \pi \oplus \rho \in \mathcal{B}_{P \cup R}^{g, g^{\prime}}, \operatorname{Res}_{s_{g P}=0}^{\operatorname{Res}} A\left(g, \pi, \rho, g^{\prime}\right) \neq 0\right\} .
$$

As shown in figure 1 , there are three ways to have non-zero residues in the $s_{g P}$ channel: the factorisation channel may have the quantum numbers of a gluon, a quark or an antiquark, the latter being permitted by a reverse orientation bracket structure. We consider them in turn 
(a)

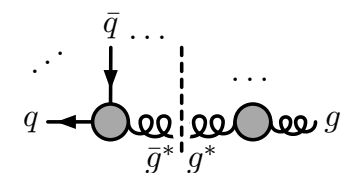

(c)

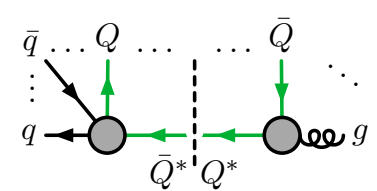

(b)

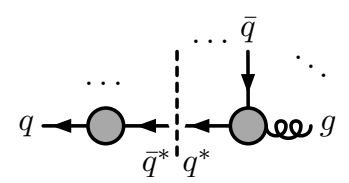

(d)

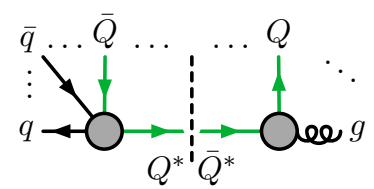

Figure 2. Factorisation channels of $q g$-basis colour-ordered amplitude.

(a) A non-zero gluonic factorisation channel $s_{g P}$ requires all quarks inside $P$ to be accompanied with the antiquarks of the same flavour, and likewise inside $R$, which guarantees no overlap between the bracket structures $\mathcal{Q}\left(F_{P}\right)$ and $\mathcal{Q}\left(F_{R}\right)$ without any further constraints. Hence, in this channel the $g g$ basis naturally factorises onto two lower-point versions of itself,

$$
\mathcal{U}_{P, R}\left[\mathcal{B}_{P \cup R}^{g, g^{\prime}}\right]=\left\{(\pi, \rho) \mid \pi \in \overline{\mathcal{Q}}\left(F_{P}\right) ш S_{G_{P}}, \quad \rho \in \overline{\mathcal{Q}}\left(F_{R}\right) \amalg S_{G_{R}}\right\}=\mathcal{B}_{P}^{g, \bar{g}^{*}} \times \mathcal{B}_{R}^{g^{*}, \bar{g}},
$$

one of which in absence of quarks in $P$ or $R$ may also be the KK basis.

(b) A valid quark factorisation channel $s_{q P}=s_{\bar{q} R}$ needs all but one quark pair to be entirely inside $P$ or $R$. Moreover, any quark lines that appear after $q$ in $\pi \in S_{P}$ or before $\bar{q}$ in $\rho \in S_{R}$ must enter in a canonical ordering, as they are enclosed in the $q \bar{q}$ bracket. This results in an exact factorisation of the $g g$ basis onto two $q g$ bases:

$$
\begin{aligned}
\mathcal{U}_{P, R}\left[\mathcal{B}_{P \cup R}^{g, g^{\prime}}\right] & =\left\{(\pi, \rho) \mid \pi \oplus\left(\bar{q}^{*}\right) \in \overline{\mathcal{Q}}\left(F_{P}\right) \amalg S_{G_{P}}, \quad\left(q^{*}\right) \oplus \rho \in \overline{\mathcal{Q}}\left(F_{R}\right) \amalg S_{G_{R}}\right\} \\
& =\mathcal{B}_{P}^{g, \bar{q}^{*}} \times \mathcal{B}_{R}^{q^{*}, g^{\prime}},
\end{aligned}
$$

where $\mathcal{B}_{P}^{g, \bar{q}^{*}}$ is a flipped version of eq. (3.38b).

(c) We remind the reader that we use the words "quark" and "antiquark" merely to label two dual representations of the gauge group (within the chosen representation labeled by "flavour"), and they can be exchanged at will within each pair. Therefore, the antiquark factorisation channel $s_{\bar{q} P}=s_{q R}$ gives $\mathcal{U}_{P, R}\left[\mathcal{B}_{P \cup R}^{g, g^{\prime}}\right]=\mathcal{B}_{P}^{g, q^{*}} \times \mathcal{B}_{R}^{\bar{q}^{*}, g^{\prime}}$ simply by relabeling the quark channel above.

Next we consider the factorised suborderings (2.9) of a $q g$ basis. Figure 2 shows its four non-vanishing channels of the form $s_{q P}=s_{g R}$ : a gluon, a quark of the same flavour as $q$, and a quark or an antiquark of another flavour. Again, the bracket structures govern the orderings that give a non-zero residue such that all lower-point combinations are consistently obtained in the limit:

$$
\begin{aligned}
\mathcal{U}_{P, R}\left[\mathcal{B}_{P \cup R}^{q, g}\right] & =\left\{(\pi, \rho) \mid(q) \oplus \pi \in \overline{\mathcal{Q}}\left(F_{P}\right) ш S_{G_{P}}, \quad \rho \in \overline{\mathcal{Q}}\left(F_{R}\right) \amalg S_{G_{R}}\right\}=\mathcal{B}_{P}^{q, \bar{g}^{*}} \times \mathcal{B}_{R}^{g^{*}, g}, \\
\mathcal{U}_{P, R}\left[\mathcal{B}_{P \cup R}^{q, g}\right] & =\left\{(\pi, \rho) \mid \pi \in \mathcal{Q}\left(F_{P}\right) ш S_{G_{P}}, \quad\left(q^{*}\right) \oplus \rho \in \overline{\mathcal{Q}}\left(F_{R}\right) \amalg S_{G_{R}}\right\}=\mathcal{B}_{P}^{q, \bar{q}^{*}} \times \mathcal{B}_{R}^{q^{*}, g}, \\
\mathcal{U}_{P, R}\left[\mathcal{B}_{P \cup R}^{q, g}\right] & =\left\{(\pi, \rho) \mid(q) \oplus \pi \oplus\left(\bar{Q}^{*}\right) \in \overline{\mathcal{Q}}\left(F_{P}\right) \amalg S_{G_{P}}, \quad\left(Q^{*}\right) \oplus \rho \in \overline{\mathcal{Q}}\left(F_{R}\right) \amalg S_{G_{R}}\right\} \\
& =\mathcal{B}_{P}^{q, \bar{Q}^{*}} \times \mathcal{B}_{R}^{Q^{*}, g} \\
\mathcal{U}_{P, R}\left[\mathcal{B}_{P \cup R}^{q, g}\right] & =\mathcal{B}_{P}^{q, Q^{*}} \times \mathcal{B}_{R}^{\bar{Q}^{*}, g} .
\end{aligned}
$$


(a)

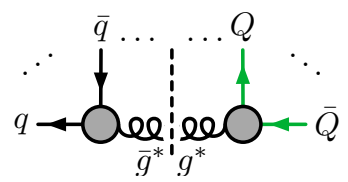

(b)

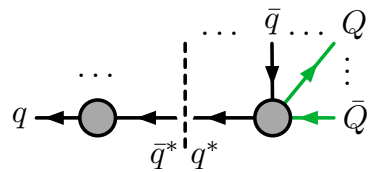

(c)

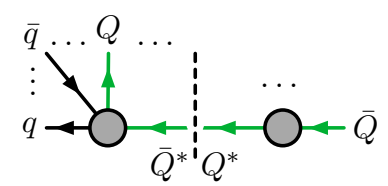

(d)

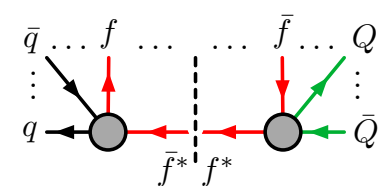

(e)

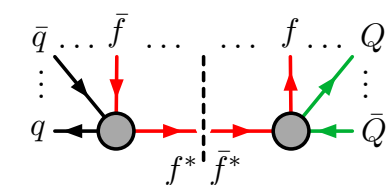

Figure 3. Factorisation channels of $q Q$-basis colour-ordered amplitude.

In particular, note how in the $q$ channel (3.42b) flavour conservation constrained the left permutation $\pi$ to bracket structures with no flips, thus giving Melia's $q q$ basis on one side and a lower-point version of the $q g$ basis on the other. The last two quark channels also factorise onto lower-point versions of the $q g$ basis on the right, while producing $q Q$ bases on the left.

Finally, a $q Q$ basis allows five non-zero factorisation limits of the form $s_{q P}=s_{Q R}$, shown in figure 3: the particle can be a gluon, a quark of either flavour $q$ or $Q$, or a quark or antiquark of a third flavour. Considering when an ordered amplitude $A(q, \pi, \rho, \bar{Q})$ gives a non-vanishing residue in these five cases, we obtain

$$
\begin{aligned}
\mathcal{U}_{P, R}\left[\mathcal{B}_{P \cup R}^{q, Q}\right] & =\left\{(\pi, \rho) \mid(q) \oplus \pi \in \overline{\mathcal{Q}}\left(F_{P}\right) \amalg S_{G_{P}}, \rho \oplus(\bar{Q}) \in \overline{\mathcal{Q}}\left(F_{R}\right) \amalg S_{G_{R}}\right\} \\
& =\mathcal{B}_{P}^{q, \bar{g}^{*}} \times \mathcal{B}_{R}^{g^{*}, Q}, \\
\mathcal{U}_{P, R}\left[\mathcal{B}_{P \cup R}^{q, Q}\right] & =\left\{(\pi, \rho) \mid \pi \in \mathcal{Q}\left(F_{P}\right) ш S_{G_{P}},\left(q^{*}\right) \oplus \rho \oplus(\bar{Q}) \in \overline{\mathcal{Q}}\left(F_{R}\right) \amalg S_{G_{R}}\right\} \\
& =\mathcal{B}_{P}^{q, \bar{q}^{*}} \times \mathcal{B}_{R}^{q^{*}, Q}, \\
\mathcal{U}_{P, R}\left[\mathcal{B}_{P \cup R}^{q, Q}\right] & =\mathcal{B}_{P}^{q, \bar{Q}^{*}} \times \mathcal{B}_{R}^{Q^{*}, Q}, \\
\mathcal{U}_{P, R}\left[\mathcal{B}_{P \cup R}^{q, Q}\right] & =\left\{(\pi, \rho) \mid(q) \oplus \pi \oplus\left(\bar{f}^{*}\right) \in \overline{\mathcal{Q}}\left(F_{P}\right) \amalg S_{G_{P}},\left(f^{*}\right) \oplus \rho \oplus(\bar{Q}) \in \overline{\mathcal{Q}}\left(F_{R}\right) \amalg S_{G_{R}}\right\} \\
& =\mathcal{B}_{P}^{q, \bar{f}^{*}} \times \mathcal{B}_{R}^{f^{*}, Q}, \\
\mathcal{U}_{P, R}\left[\mathcal{B}_{P \cup R}^{q, Q}\right] & =\mathcal{B}_{P}^{q, f^{*}} \times \mathcal{B}_{R}^{\bar{f}^{*}, Q} .
\end{aligned}
$$

\subsubsection{Colour-ordered splitting}

Here we show that the bases of colour-ordered amplitudes described in this section indeed satisfy the "colour-ordered splitting" relation, equation (2.14). In words this requires us to show that all elements of an $n$-point basis with particle $i$ before particle $j$ are exactly those found when taking an $(n-1)$-point basis and performing all possible ordered splittings producing this pair. As all bases contain each splitting, we consider each limit in turn.

$\boldsymbol{q g}$ and $\boldsymbol{g g}$ splitting. If one of particles $i$ and $j$ is a gluon, then we can easily show that all bases respect the ordered splitting. Note that the associated $(n-1)$-point basis is that with one fewer gluon. Importantly, the $n$-point basis can then be thought of as inserting the remaining gluon in all possible positions in the $(n-1)$-point basis. This necessarily 
includes an insertion adjacent to the other particle and so the colour-ordered splitting relation (2.14) is satisfied. Comparing eqs. (2.11) and (2.12) we obtain the colour-factor identity (2.4a) in the $q g$ case and relation (2.5) in the $g g$ case.

$\boldsymbol{q} \overline{\boldsymbol{q}}$ splitting. The remaining case is more complicated, as for the $q \bar{q}$ splitting certain terms in the derivation, specifically in eq. (2.12), may be vanishing. This arises due to a property of the allowed quark brackets in the co-unitary bases. Specifically, all enclosed quark brackets only occur in one orientation, familiar from the Melia basis. In this splitting, a $q \bar{q}$ pair is necessarily produced from a gluon. Given that it respects the orientation criteria, an insertion of an adjacent quark pair $\{q, \bar{q}\}$ is as legal as a single gluon insertion in all bases. Indeed, the consistency of a quark bracket structure is never disturbed by either, so the elements of the two bases are in one-to-one correspondence as required in eq. (2.14).

If the splitting gluon is found in an enclosed position, we end up with only one term when equating eqs. (2.11) and (2.12). Considering Melia's basis, this holds for the colour factors (3.9) by construction. If the splitting corresponds to an unenclosed gluon, then we end up with both terms. This is then the origin of the $\theta$ in the colour-factor identity (2.3).

\subsubsection{Linear independence}

Finally, let us now demonstrate the linear independence of the sets of ordered amplitudes proposed in this work. This is sufficient to show that these sets form bases, given that the sets have the right counting $(n-2) ! / k$ !. To simplify the argumentation, we further assume the linear independence of the Melia basis as established by refs. [26, 27].

First, we note that all three-particle amplitude bases are trivially independent as they involve only one element. We wish to work inductively, assuming that for all $m<n$ our sets of $m$-point amplitudes form bases of their respective kinematic space. Precisely, we assume that the only values of $\alpha_{\chi}$ that solve the equation

$$
\sum_{\chi \in \mathcal{B}_{X}^{a, b}} \alpha_{\chi} A(\stackrel{\chi}{\underbrace{\chi}})=0
$$

where all amplitudes are $m$-point, are the trivial solutions $\alpha_{\chi}=0$. Now we look to find possible solutions $\alpha_{\sigma}$ of the equation

$$
\sum_{\sigma \in \mathcal{B}_{P \cup R}^{1, n}} \alpha_{\sigma} A\left(1 \stackrel{\sigma}{\ddots}{ }_{n}\right)=0,
$$

where we consider the pair of stretched particles $(1, n)$ to be anything other than $(q, \bar{q})$. To find solutions, we once again take a factorisation limit that separates the particles into the two sets $\{1\} \cup P$ and $R \cup\{n\}$. Importantly, we note that each $\alpha_{\sigma}$ must turn up at least in some limit of this form, as every colour-ordered amplitude contains at least one channel in which it has a non-vanishing residue. (For this reason we rely on the independence of the Melia basis: it contains ordered amplitudes that do not have such factorisation channels.) 
By co-unitarity of our amplitude sets, such a limit results in

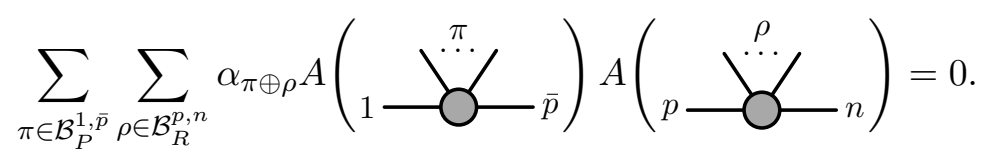

As our induction hypothesis states that there are no relations between $A(1, \pi, \bar{p})$, the coefficient of each ordered amplitude must vanish independently, so we have

$$
\sum_{\rho \in \mathcal{B}_{R}^{p, n}} \alpha_{\pi \oplus \rho} A\left(\stackrel{\rho}{\rho^{\cdots}}\right)=0
$$

for each permutation $\pi$. However, again by hypothesis, the only solutions to this equation are $\alpha_{\pi \oplus \rho}=0$. As this works for all possible limits, this shows that the coefficients $\alpha_{\sigma}$ in eq. (3.45) are indeed all zero, and so the elements are linearly independent.

In summary, we have constructed amplitude sets with the correct number of elements that satisfy the factorisation property (2.2). This guarantees that the sets are valid bases.

\section{Loop-level applications}

In ref. [20] we presented a general full-colour construction for loop amplitudes in Yang-Mills theory from generalised unitarity cuts, which had been used in the two-loop calculation of ref. [14]. In this section we outline the extension of this construction to QCD.

The master formula stays the same as in ref. [20]:

$$
\mathcal{A}_{n}^{(L)}=i^{L-1} \sum_{i \in \text { KK-indep. 1PI graphs }} \int \frac{d^{L D} \ell}{(2 \pi)^{L D}} \frac{C_{i} \Delta_{i}}{S_{i} \prod_{l \in i} D_{l}} .
$$

The summation here is over the topologies of the ordered unitarity cuts, from which the kinematic numerators $\Delta_{i}$ are obtained [36-41]. The cuts are constructed with tree-level amplitudes as vertices, and for each such tree only a set of KK-independent orderings need be retained. The denominators involve the graph symmetry factors $S_{i}$ (calculated in the unordered sense) and the propagator denominators $D_{l}$ that were put on shell to compute the cuts and therefore the numerators $\Delta_{i}$. These numerators may contain poles with respect to the external momenta but only polynomial dependence on the loop momenta.

The crucial content of the above formula is the specification that the colour factors $C_{i}$ are exactly inherited from the tree-level colour decompositions inside the unitarity cuts. This is a simple solution to the a priori puzzling question of how to combine the physical information from unitarity cuts into a full-colour integrand without double counting.

In the purely gluonic case the formula (4.1) means that one may fix any two edges of every vertex and sum only over the permutations of the remaining edges, such that the colour structures inside the vertex are given by the comb-like colour structures $C_{\mathrm{DDM}}\left(g, \sigma, g^{\prime}\right)$ "stretched" by the fixed edges. The choice of which two edges to fix is a priori arbitrary as this will always result in KK independent sets. However, in ref. [20] we show that this freedom can be exploited to further simplify the loop-amplitude construction with "stretch" choices tailored to specific loop topologies. 


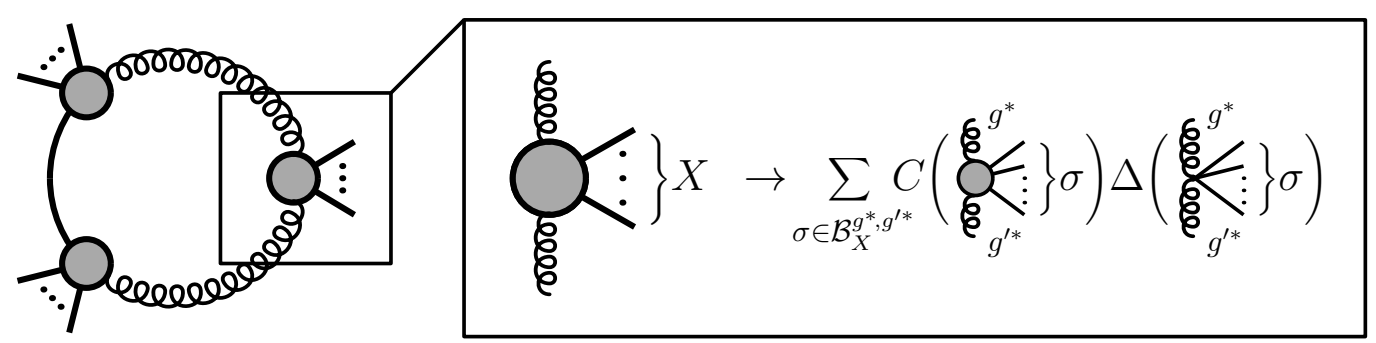

Figure 4. Inserting a gluon-gluon stretch tree basis into coloured cuts of a one-loop amplitude.

In this paper we have formulated a complete set of KK-independent bases and colour decompositions for QCD tree amplitudes, which now allow a similarly flexible application of the construction (4.1) to loop amplitudes in QCD (or similar gauge theories with matter). That is, at any stage of a unitarity-based calculation only the KK-independent cut orderings need be considered, which correspond to arbitrarily chosen "stretches" by two edges of the cut diagram vertices. The resulting numerators are then dressed with the colour structures that are sewn from comb-like structures with occasional $C_{\mathrm{JO}}(q, \sigma, \bar{q})$ elements.

One-loop colour decomposition. Let us now illustrate the full-colour approach at one-loop for QCD amplitudes with external quarks. Without loss of generality, we restrict to the case of all external quark pairs having different flavours, as all other cases may be computed by antisymmetrisation over the distinct-flavour case. For example, an $n$-point amplitude with four identical quarks can be expressed, irrespectively of the loop order, as

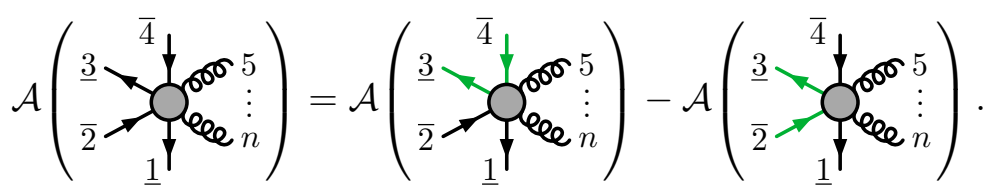

Here the distinct-flavour amplitudes on the right-hand side are taken with equal quark masses but with a relative sign, implementing the fermionic antisymmetry of the likeflavour amplitude on the left-hand side.

Working in $D=4-2 \epsilon$ dimensions, the one-particle irreducible topologies that one should consider in eq. (4.1) at one loop have up to five vertices, each with three or more edges. Two of these edges are loop-momentum dependent, to which we shall refer as "loop edges" and the rest correspond to the external particles. For each such topology, one then dresses it in all possible ways with full-colour tree amplitudes to find the set of possible unordered unitarity cuts. From these diagrams we compute the symmetry factor $S_{i}$ in eq. (4.1). For each diagram, one can now make a choice of the associated set of KK-independent ordered unitarity cut diagrams that are summed over in eq. (4.1) and correspond to the numerators $\Delta_{i}$. In this one-loop case, we choose to stretch the constituent tree amplitudes across the loop edges, which specifies the KK-independent basis of each corner to be the ones of this paper. This choice brings two advantages. First, none of the ordered topologies have legs pointing inside the loop, and so all numerators can be readily associated with the leading-colour ordered amplitudes. Secondly, all kinematic factorisation limits which intertwine the loop-dependent numerators are also respected by 


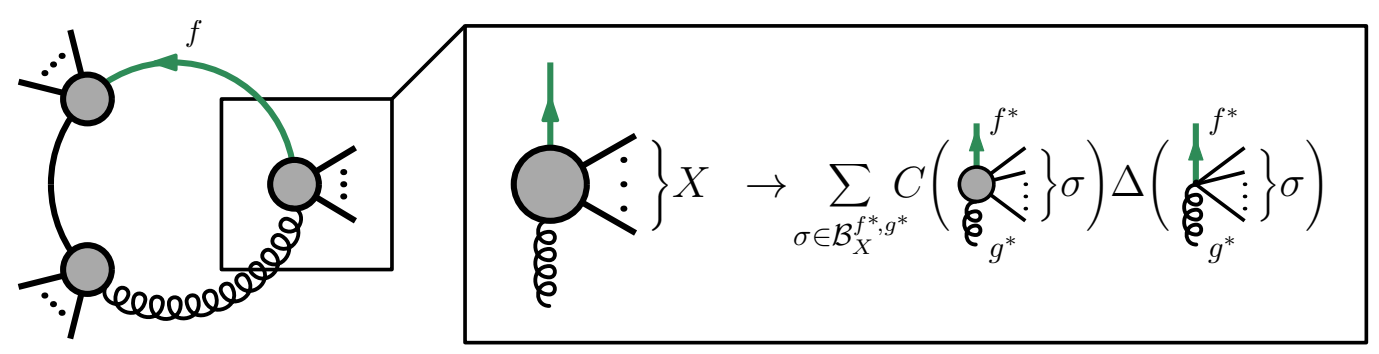

Figure 5. Inserting a quark-gluon stretch tree basis into coloured cuts of a one-loop amplitude.

the colour factor through the colour factorisation relation (2.2). Therefore, all colourordered numerators related by factorisation come with the same colour factor.

Let us now consider how this procedure captures the combinatorics of multi-quark one-loop amplitudes. In contrast to the purely gluonic case, there are two novelties. The most evident is that the two "loop edges" across which the tree amplitudes are stretched can now correspond to any two different particles in the theory. The second is that, due to internal quarks running inside the loop, the tree amplitudes in the vertices cannot be reduced to distinct flavour amplitudes. We shall work through these details by considering each possible type of stretch in turn. For every vertex inside a cut, its loop edges may correspond to either

- two internal gluons, as depicted in figure 4;

- one quark and one gluon, illustrated in figure 5;

- two internal quarks, as shown in figure 6 for the case of the like-flavour edges.

In order to concretely discuss the details we discuss these three cases using the example of two-particle cuts of an $n$-point two-quark amplitude at one loop. Similar to the adjoint case of ref. [20], we shall see how the symmetry factors cancel in the construction. To do this, we first organise the contributions to eq. (4.1) into colour-dressed numerators corresponding to an unordered graph; we denote such numerators by $\tilde{\Delta}_{i}$.

Consider an $s_{12}$-channel bubble topology, in which a purely gluonic loop is exposed. The symmetry factor of this bubble is 2 . The coloured numerator is given by

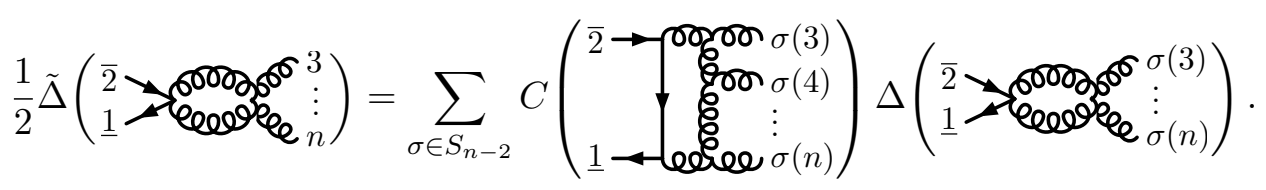

The set of permutations over which one sums in each corner is given by the bases with two fixed gluon legs. In the concrete example of eq. (4.3), the explicit permutations and the colour factors on the right-hand side of the cut are dictated by the DDM decomposition (1.1). The colour factor on the left-hand side at this point is also a simple comb-like structure involving both quarks. The sum over the permutations $\underline{1} \leftrightarrow \overline{2}$ on the left naturally produces two copies of each colour-ordered numerator, which when considered under the integral sign cancel the symmetry factor 2 . This may be familiar from the purely gluonic 


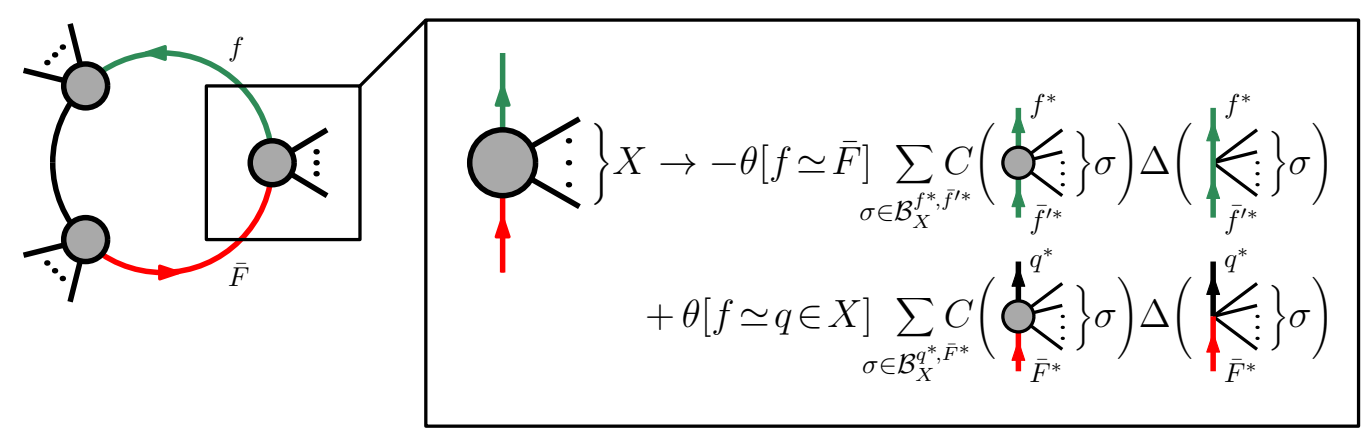

Figure 6. Inserting a quark-quark stretch tree basis into coloured cuts of a one-loop amplitude. The $\theta$ functions are non-zero when the two flavours are the same. The first term is present if the stretched quarks are of the same flavour. The second term is present if the stretched flavour is also in the set of external flavours.

case [20]. This property relies on the permutation sum generating two copies of each term related only by a reflection across the axis of the bubble. For this to hold in more general cases with multiple quark lines on either side, one must take care to use a basis with a quark bracket signature such that the basis is invariant under the exchange [] $\leftrightarrow\{\}{ }^{8}$

Next, consider a bubble cut which involves two distinct gluonic and fermionic loop lines, whose symmetry factor is unity. Flavour conservation implies that the flavour of the internal quark line coincides with an external quark pair split by the unitarity cut. For such a bubble in the two-quark amplitude, the colour decomposition presented in figure 5 should be applied to both sides of the cut. Schematically, this gives

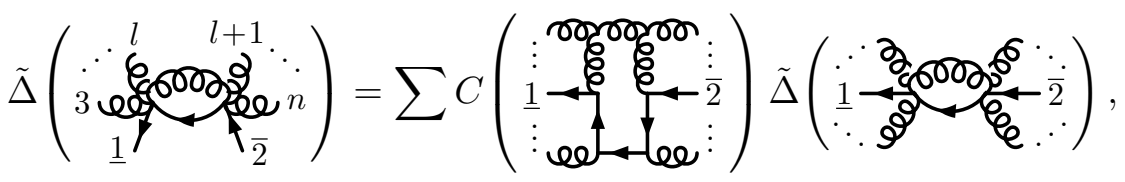

where the sum goes over all permutations of the left- and right-hand particle sets. Due to the absence of differently flavoured quark lines, the loop colour factors above are sewn from two comb structures, which are a special case of the $q g$ colour factors (3.28).

Finally, let us consider the case where both loop legs are fermionic, where we need to employ the tactics of figure 6 . Here we face the subtlety where the external and internal fermion lines may indeed be of the same flavour. For a concrete example we choose the quark-loop version of the $s_{12}$-channel bubble topology. We shall label the flavour of the quark loop by $f$ which may or may not be the same flavour as the external line. In this

\footnotetext{
${ }^{8}$ In section 3.2 and 3.3, when we allowed the unnested quark brackets to come in both combinations \{\} and [], we chose the signature for all the nested quark brackets to remain canonical. This convention may be switched to have all the nested brackets follow the signature of their enclosing bracket, i.e. $[\ldots\{\ldots\} \ldots] \rightarrow$ $[\ldots[\ldots] \ldots]$. At one loop, this choice allows the colour decompositions to respect the reflection invariance of the bubbles with internal gluons. This convention is also consistent with ref. [33].
} 
case we can write the colour-dressed numerator as

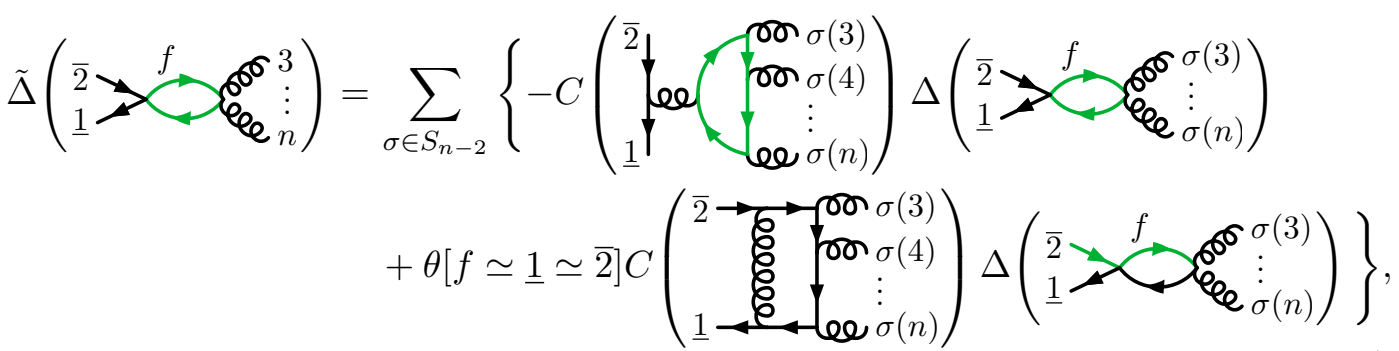

where the theta function encodes the fact that the second term only contributes if $f$ is the same flavour as particles $\underline{1}$ and $\overline{2}$. As the two internal quark lines are distinct, the symmetry factor is unity. The contributions in the first line of eq. (4.5) enter because the loop edges are two quarks of the same flavour $f$. The gluonic permutations on the right-hand side of the bubble generate simple DDM comb-like colour factors. The left-hand side of the bubble does not require any permutations due to the fixed quark signature of the four-quark Melia basis, and the corresponding colour factor is familiar from eq. (1.4). The second line of eq. (4.5) only contributes if the internal quarks are of the same flavour as the external ones, in accord with the construction (4.2) of the like-flavour amplitude from the distinct-flavour ones. The fixed quark signature of $q Q$-stretch basis on the left-hand side of the bubble still requires no permutations, apart from the gluonic ones from the right-hand side. The colour factors are comb-like on both sides due to the absence of additional quark lines.

Of particular interest is the relative fermionic sign between the two lines of eq. (4.5). The first line corresponds to the so-called $N_{f}$ contributions to the amplitude due to a closed quark loop, whereas in the second line the quark loop is not closed. Indeed, the associated unitarity cut contains a loop-momentum dependent channel that corresponds to a gluonic line. Due to colour factorisation, this is already manifest in the colour factor. We observe that the resulting (relative) fermionic sign follows through from the flavour-permutation construction (4.2). It is a general feature of our approach that the fermionic signs from flavour permutations feed into unitarity cuts and produce consistent signs for topologies with fermion loops.

In the above examples, we have reduced the colour factors to explicit diagrams with only three-point vertices. However, their more general feature is that they are all given by a diagram which is a ring built from the $C_{\mathrm{DDM}}\left(g, \sigma, g^{\prime}\right)$ and $C_{\mathrm{JO}}(q, \sigma, \bar{q})$ building blocks. We emphasise that this is a general feature of the one-loop decomposition, enabled by the colour factorisation relation (2.2) of the tree-level decompositions. As the constituent tree amplitudes in each cut are stretched across the loop legs, and the tree colour factors themselves factorise into such building blocks, this construction is naturally inherited by the loop-level factors.

Finally, we note that the presented approach is completely consistent with the known methods for colour decompositions of multi-quark amplitudes. For example, the colourordered numerators that we have constructed here can be associated with the left/rightturner and $N_{f}$ families of one-loop primitive amplitudes defined in refs. [29, 30]. Furthermore, our results are also completely consistent with Kälin's recent one-loop decomposition 
into such primitive amplitudes [33], as can be easily verified by comparing the unitarity cuts. However, the tree-level results cannot be directly derived from Kälin's decomposition, as it does not involve symmetry factors and so its cuts correspond to products of tree-level amplitudes with certain terms identified.

\section{Summary and outlook}

In this paper we have considered the colour structure of tree and one-loop QCD amplitudes involving any number of distinctly flavoured quark-antiquark pairs. At tree level, we have derived new bases of ordered amplitudes that are independent under the KleissKuijf relations [23] and found decompositions of an $n$-point colour-dressed amplitude into these bases. In combination with the previously known colour decompositions of refs. [21] and [24, 25], our results permit flexible amplitude implementations in terms of ordered amplitudes with an arbitrary pair of partons fixed next to each other. At (multi-) loop level, this flexibility significantly enhances the application of the loop-colour approach of ref. [20], as demonstrated by our more detailed exposition at one loop.

Another important aspect of this paper is the recursive unitarity-based approach that we employ to construct new colour decompositions. It relies on the physical factorisation properties of the colour-dressed and colour-ordered amplitudes, which impose certain factorisation relations on the colour factors. Due to its physical transparency, this method for handling colour factors could arguably be considered as advantageous with respect to closed-form expressions. For instance, the implementation strategy where colour factors are recursed down to fundamental three-point vertices has already proven its simplicity in the recent two-loop computations at leading colour $[10,12]$ by one of the authors.

Apart from the proposed utility of our results for future multi-loop QCD calculations via on-shell methods, there are a number of other natural applications. In particular, the colour-kinematics duality [34, 42] present in QCD [24, 43] can be used to uplift the presented colour decompositions to new representations of gravitational amplitudes in QCD minimally coupled to general relativity [44]. Moreover, the duality implies the kinematicdependent amplitude relations [24, 34, 45], which could be used to further reduce the bases formulated here. It could be interesting to explore whether such reduced bases could provide additional flexibility in the application of such amplitude relations to loop amplitudes [14, 46, 47].

Our loop-colour approach [20] is a tool for constructing the loop integrand in gauge theory. The colour factorisation perspective taken in this paper naturally organises loop integrand contributions in this approach, as we have explicitly demonstrated at one loop. However, there are a number of available analytical full-colour results for two-loop amplitudes in pure Yang-Mills theory [13], its $\mathcal{N}=4$ supersymmetric extension [48-50] and $\mathcal{N}=2$ supersymmetric QCD [51], which organise the integrated amplitudes in terms of the more traditional trace basis, subject to known colour redundancies [52]. A natural question is then how factorisation could guide the organisation of colour structures, not only of the integrand of loop amplitudes, but also after integration. 


\section{Acknowledgments}

We thank Samuel Abreu, Harald Ita and Henrik Johansson for helpful conversations. We also thank Fernando Febres Cordero, Henrik Johansson and Gregor Kälin for comments on the manuscript. AO's research is funded by the European Union's Horizon 2020 research and innovation programme under the Marie Skłodowska-Curie grant agreement 746138 . BP is grateful for the support from the Pauli Center for Theoretical Physics. The work of B.P. is supported by the French Agence Nationale pour la Recherche, under grant ANR17-CE31-0001-01.

\section{A Colour Feynman rules and colour ordering}

Here we recall the standard colour vertices defined with respect to planar ordering:

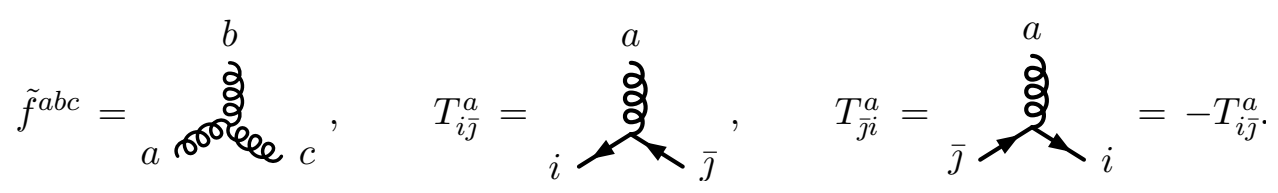

A convenient way to normalise the gauge-group generators is by imposing

$$
\operatorname{Tr}\left(T^{a} T^{b}\right)=\delta^{a b}, \quad\left[T^{a}, T^{b}\right]=\tilde{f}^{a b c} T^{c} \quad \Rightarrow \quad \tilde{f}^{a b c}=\operatorname{tr}\left(\left[T^{a}, T^{b}\right] T^{c}\right) .
$$

The purely kinematic ordered Feynman rules may be found e.g. in ref. [22] but their specific form is irrelevant for the purely colour-algebraic results of the present paper. The general applicability of the concept of colour ordering relies on the fact that in a unitary gauge theory a matter particle in a complex group representation can be projected to two real particles in the adjoint representations with the same kinematic Feynman diagrams but with all generators replaced by the structure constants. Colour ordering can then be performed in the same way as for gluons, thereby defining the ordered amplitudes $A(\sigma(1), \ldots, \sigma(n))$ with matter as gauge-invariant kinematic coefficients of the fundamental traces $\operatorname{tr}\left(T^{a_{\sigma(1)}} \cdots T^{a_{\sigma(n)}}\right)$. Once ordered in this way in the adjoint representation, these amplitudes can be dressed with the arbitrary-representation colour coefficients, on which we concentrate in the bulk of the paper.

\section{B Colour-unitarity checks}

Here we show that the colour factors given by eq. (3.9) obey the leg-exchange relations (2.3), (2.4) and (2.5). The former is satisfied by construction as illustrated by the first line of eq. (3.8): when a quark pair is not separated by any other particles, its colour factor factorises explicitly onto the adjoint index of the intermediate gluon via $\left\{q\left|T^{a_{g *}}\right| \bar{q}\right\}=T_{i_{q} \bar{q}_{\bar{q}}}^{a_{g *}}$.

The gluonic colour-factor relation (2.5) is also obeyed automatically due to the commutation relation between the tensor-representation generators,

$$
\left[\Xi_{l}^{a}, \Xi_{l}^{b}\right]=\tilde{f}^{a b c} \Xi_{l}^{c}
$$


Indeed, when two gluons are next to each other, the corresponding colour factor necessarily involves two $\Xi$ 's of the same nestedness level $l$, so the commutation relation generates the adjoint vertex to the intermediate gluon, as in eq. (2.5).

Now let us consider the quark-gluonic exchange relation (2.4) in detail. Drawing only the relevant parts of the colour diagrams for the $q g$ case, we compute in full generality
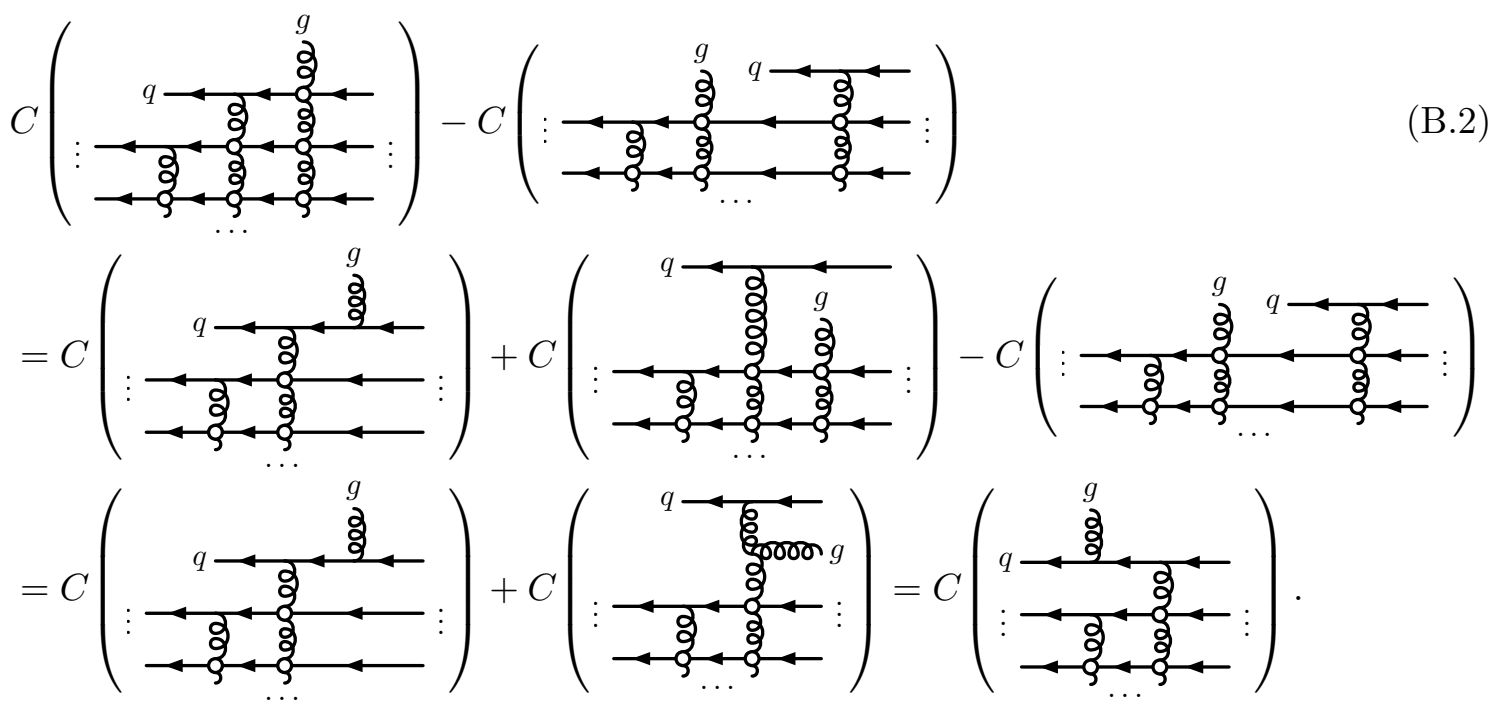

Here we first used the additive nature of the tensor-representation generators that is evident from the definition, namely $\Xi_{l}^{a}=T_{l}^{a}+\Xi_{l-1}^{a}$, and then applied the commutation relations (B.1) and (2.6b). In the resulting diagram the original quark $q$ and gluon $g$ are attached to a lower-point colour factor of the type (3.9) in exactly the same way as required by the leg-exchange identity (2.4a). Finally, the corresponding $g \bar{q}$ condition (2.4b) follows exclusively from the aforementioned recurrence relation $\Xi_{l}^{a}=T_{l}^{a}+\Xi_{l-1}^{a}$ :

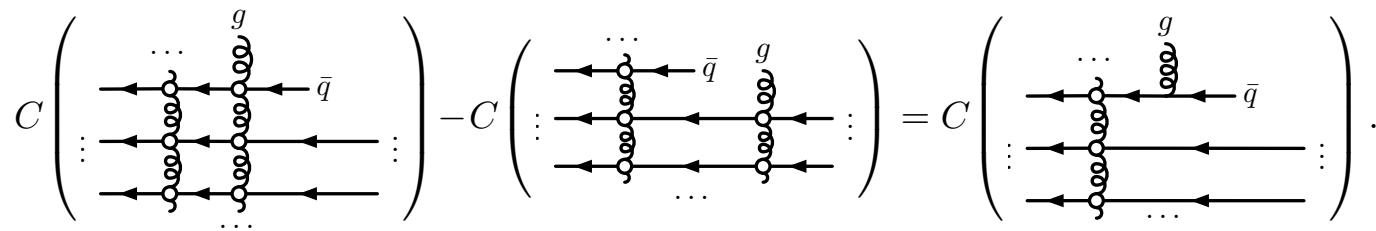

In other words, the left-right asymmetric choice of the colour diagrams in eq. (3.9) makes the $g \bar{q}$-exchange property (2.4b) manifest for the price making the $q g$-exchange property $(2.4 \mathrm{a})$ hidden behind a couple of commutation relations.

Open Access. This article is distributed under the terms of the Creative Commons Attribution License (CC-BY 4.0), which permits any use, distribution and reproduction in any medium, provided the original author(s) and source are credited.

\section{References}

[1] T. Gehrmann, J.M. Henn and N.A. Lo Presti, Analytic form of the two-loop planar five-gluon all-plus-helicity amplitude in QCD, Phys. Rev. Lett. 116 (2016) 062001 [Erratum ibid. 116 (2016) 189903] [arXiv: 1511.05409] [INSPIRE]. 
[2] D.C. Dunbar and W.B. Perkins, Two-loop five-point all plus helicity Yang-Mills amplitude, Phys. Rev. D 93 (2016) 085029 [arXiv:1603.07514] [InSPIRE].

[3] D.C. Dunbar, G.R. Jehu and W.B. Perkins, The two-loop n-point all-plus helicity amplitude, Phys. Rev. D 93 (2016) 125006 [arXiv:1604.06631] [INSPIRE].

[4] D.C. Dunbar, G.R. Jehu and W.B. Perkins, Two-loop six gluon all plus helicity amplitude, Phys. Rev. Lett. 117 (2016) 061602 [arXiv:1605.06351] [INSPIRE].

[5] D.C. Dunbar, J.H. Godwin, G.R. Jehu and W.B. Perkins, Analytic all-plus-helicity gluon amplitudes in QCD, Phys. Rev. D 96 (2017) 116013 [arXiv:1710.10071] [InSPIRE].

[6] S. Badger, C. Bronnum-Hansen, H.B. Hartanto and T. Peraro, First look at two-loop five-gluon scattering in QCD, Phys. Rev. Lett. 120 (2018) 092001 [arXiv:1712.02229] [INSPIRE].

[7] S. Abreu et al., Planar two-loop five-gluon amplitudes from numerical unitarity, Phys. Rev. D 97 (2018) 116014 [arXiv:1712.03946] [INSPIRE].

[8] H.A. Chawdhry, M.A. Lim and A. Mitov, Two-loop five-point massless QCD amplitudes within the integration-by-parts approach, Phys. Rev. D 99 (2019) 076011 [arXiv: 1805.09182] [INSPIRE].

[9] S. Badger et al., Applications of integrand reduction to two-loop five-point scattering amplitudes in QCD, PoS (LL2018) 006 [arXiv: 1807.09709] [INSPIRE].

[10] S. Abreu et al., Planar two-loop five-parton amplitudes from numerical unitarity, JHEP 11 (2018) 116 [arXiv: 1809.09067] [INSPIRE].

[11] S. Abreu et al., Analytic form of planar two-loop five-gluon scattering amplitudes in QCD, Phys. Rev. Lett. 122 (2019) 082002 [arXiv: 1812.04586] [INSPIRE].

[12] S. Abreu et al., Analytic form of the planar two-loop five-parton scattering amplitudes in QCD, JHEP 05 (2019) 084 [arXiv: 1904.00945] [INSPIRE].

[13] S. Badger et al., Analytic form of the full two-loop five-gluon all-plus helicity amplitude, Phys. Rev. Lett. 123 (2019) 071601 [arXiv:1905.03733] [InSPIRE].

[14] S. Badger, G. Mogull, A. Ochirov and D. O'Connell, A complete two-loop, five-gluon helicity amplitude in Yang-Mills theory, JHEP 10 (2015) 064 [arXiv:1507.08797] [INSPIRE].

[15] S. Badger, H. Frellesvig and Y. Zhang, A two-loop five-gluon helicity amplitude in QCD, JHEP 12 (2013) 045 [arXiv: 1310.1051] [INSPIRE].

[16] S. Badger, G. Mogull and T. Peraro, Local integrands for two-loop all-plus Yang-Mills amplitudes, JHEP 08 (2016) 063 [arXiv: 1606.02244] [INSPIRE].

[17] Z. Bern, L.J. Dixon, D.C. Dunbar and D.A. Kosower, One loop $n$ point gauge theory amplitudes, unitarity and collinear limits, Nucl. Phys. B 425 (1994) 217 [hep-ph/9403226] [INSPIRE].

[18] Z. Bern, L.J. Dixon, D.C. Dunbar and D.A. Kosower, Fusing gauge theory tree amplitudes into loop amplitudes, Nucl. Phys. B 435 (1995) 59 [hep-ph/9409265] [InSPIRE].

[19] R. Britto, F. Cachazo and B. Feng, Generalized unitarity and one-loop amplitudes in $N=4$ Super-Yang-Mills, Nucl. Phys. B 725 (2005) 275 [hep-th/0412103] [INSPIRE].

[20] A. Ochirov and B. Page, Full colour for loop amplitudes in Yang-Mills theory, JHEP 02 (2017) 100 [arXiv:1612.04366] [INSPIRE]. 
[21] V. Del Duca, L.J. Dixon and F. Maltoni, New color decompositions for gauge amplitudes at tree and loop level, Nucl. Phys. B 571 (2000) 51 [hep-ph/9910563] [INSPIRE].

[22] L.J. Dixon, Calculating scattering amplitudes efficiently, in the proceedings of the Theoretical Advanced Study Institute in Elementary Particle Physics (TASI-95), June 4-30, Boulder, U.S.A. (1995), hep-ph/9601359 [inSPIRE].

[23] R. Kleiss and H. Kuijf, Multi-gluon cross-sections and five jet production at hadron colliders, Nucl. Phys. B 312 (1989) 616 [inSPIRE].

[24] H. Johansson and A. Ochirov, Color-kinematics duality for QCD amplitudes, JHEP 01 (2016) 170 [arXiv:1507.00332] [INSPIRE].

[25] T. Melia, Proof of a new colour decomposition for QCD amplitudes, JHEP 12 (2015) 107 [arXiv: 1509.03297] [INSPIRE].

[26] T. Melia, Dyck words and multiquark primitive amplitudes, Phys. Rev. D 88 (2013) 014020 [arXiv: 1304.7809] [INSPIRE].

[27] T. Melia, Getting more flavor out of one-flavor QCD, Phys. Rev. D 89 (2014) 074012 [arXiv:1312.0599] [INSPIRE].

[28] Z. Bern and D.A. Kosower, Color decomposition of one loop amplitudes in gauge theories, Nucl. Phys. B 362 (1991) 389 [inSPIRE].

[29] Z. Bern, L.J. Dixon and D.A. Kosower, One loop corrections to two quark three gluon amplitudes, Nucl. Phys. B 437 (1995) 259 [hep-ph/9409393] [INSPIRE].

[30] H. Ita and K. Ozeren, Colour decompositions of multi-quark one-loop QCD amplitudes, JHEP 02 (2012) 118 [arXiv:1111.4193] [INSPIRE].

[31] C. Reuschle and S. Weinzierl, Decomposition of one-loop QCD amplitudes into primitive amplitudes based on shuffle relations, Phys. Rev. D 88 (2013) 105020 [arXiv:1310.0413] [INSPIRE].

[32] T. Schuster, Color ordering in QCD, Phys. Rev. D 89 (2014) 105022 [arXiv:1311.6296] [INSPIRE].

[33] G. Kälin, Cyclic Mario worlds - Color-decomposition for one-loop QCD, JHEP 04 (2018) 141 [arXiv: 1712.03539] [INSPIRE].

[34] Z. Bern, J.J.M. Carrasco and H. Johansson, New relations for Gauge-Theory amplitudes, Phys. Rev. D 78 (2008) 085011 [arXiv: 0805.3993] [inSPIRE].

[35] R.W. Brown and S.G. Naculich, Color-factor symmetry and BCJ relations for QCD amplitudes, JHEP 11 (2016) 060 [arXiv:1608.05291] [INSPIRE].

[36] G. Ossola, C.G. Papadopoulos and R. Pittau, Reducing full one-loop amplitudes to scalar integrals at the integrand level, Nucl. Phys. B 763 (2007) 147 [hep-ph/0609007] [InSPIRE].

[37] P. Mastrolia and G. Ossola, On the integrand-reduction method for two-loop scattering amplitudes, JHEP 11 (2011) 014 [arXiv:1107.6041] [INSPIRE].

[38] S. Badger, H. Frellesvig and Y. Zhang, Hepta-cuts of two-loop scattering amplitudes, JHEP 04 (2012) 055 [arXiv: 1202. 2019] [INSPIRE].

[39] Y. Zhang, Integrand-level reduction of loop amplitudes by computational algebraic geometry methods, JHEP 09 (2012) 042 [arXiv: 1205.5707] [INSPIRE]. 
[40] P. Mastrolia, E. Mirabella, G. Ossola and T. Peraro, Scattering amplitudes from multivariate polynomial division, Phys. Lett. B 718 (2012) 173 [arXiv:1205.7087] [INSPIRE].

[41] H. Ita, Two-loop integrand decomposition into master integrals and surface terms, Phys. Rev. D 94 (2016) 116015 [arXiv: 1510.05626] [INSPIRE].

[42] Z. Bern, J.J.M. Carrasco and H. Johansson, Perturbative quantum gravity as a double copy of gauge theory, Phys. Rev. Lett. 105 (2010) 061602 [arXiv:1004.0476] [INSPIRE].

[43] H. Johansson and A. Ochirov, Pure gravities via color-kinematics duality for fundamental matter, JHEP 11 (2015) 046 [arXiv: 1407.4772] [INSPIRE].

[44] J. Plefka and W. Wormsbecher, New relations for graviton-matter amplitudes, Phys. Rev. D 98 (2018) 026011 [arXiv: 1804.09651] [INSPIRE].

[45] L. de la Cruz, A. Kniss and S. Weinzierl, Proof of the fundamental BCJ relations for QCD amplitudes, JHEP 09 (2015) 197 [arXiv: 1508.01432] [INSPIRE].

[46] A. Primo and W.J. Torres Bobadilla, BCJ identities and d-dimensional generalized unitarity, JHEP 04 (2016) 125 [arXiv: 1602.03161] [INSPIRE].

[47] A. Ochirov, P. Tourkine and P. Vanhove, One-loop monodromy relations on single cuts, JHEP 10 (2017) 105 [arXiv:1707.05775] [INSPIRE].

[48] J.J. Carrasco and H. Johansson, Five-point amplitudes in $N=4$ Super-Yang-Mills theory and $N=8$ supergravity, Phys. Rev. D 85 (2012) 025006 [arXiv:1106.4711] [InSPIRE].

[49] S. Abreu, L.J. Dixon, E. Herrmann, B. Page and M. Zeng, The two-loop five-point amplitude in $\mathcal{N}=4$ Super-Yang-Mills theory, Phys. Rev. Lett. 122 (2019) 121603 [arXiv:1812.08941] [INSPIRE].

[50] D. Chicherin et al., Analytic result for a two-loop five-particle amplitude, Phys. Rev. Lett. 122 (2019) 121602 [arXiv:1812.11057] [INSPIRE].

[51] C. Duhr et al., The full-color two-loop four-gluon amplitude in $\mathcal{N}=2$ super-QCD, arXiv: 1904.05299 [INSPIRE].

[52] A.C. Edison and S.G. Naculich, SU $(N)$ group-theory constraints on color-ordered five-point amplitudes at all loop orders, Nucl. Phys. B 858 (2012) 488 [arXiv:1111.3821] [INSPIRE]. 